\title{
Expanding the Natural Laminar Flow Boundary for Supersonic Transports
}

\author{
Michelle N. Lynde ${ }^{1}$ and Richard L. Campbell ${ }^{2}$ \\ NASA Langley Research Center, Hampton, VA, 23681
}

\begin{abstract}
A computational design and analysis methodology is being developed to design a vehicle that can support significant regions of natural laminar flow (NLF) at supersonic flight conditions. The methodology is built in the CDISC design module to be used in this paper with the flow solvers Cart3D and USM3D, and the transition prediction modules BLSTA3D and LASTRAC. The NLF design technique prescribes a target pressure distribution for an existing geometry based on relationships between modal instability wave growth and pressure gradients. The modal instability wave growths (both on- and off-axes crossflow and Tollmien-Schlichting) are balanced to produce a pressure distribution that will have a theoretical maximum NLF region for a given streamwise wing station. An example application is presented showing the methodology on a generic supersonic transport wingbody configuration. The configuration has been successfully redesigned to support significant regions of NLF (approximately $40 \%$ of the wing upper surface by surface area). Computational analysis predicts NLF with transition Reynolds numbers $\left(\operatorname{Re}_{\mathrm{T}}\right)$ as high as 36 million with 72 degrees of leading-edge sweep $\left(\Lambda_{L E}\right)$, significantly expanding the current boundary of $\operatorname{Re}_{\mathrm{T}}-\Lambda_{\mathrm{LE}}$ combinations for NLF. This NLF geometry provides a total drag savings of 4.3 counts compared to the baseline wing-body configuration (approximately $5 \%$ of total drag). Off-design evaluations at near-cruise and low-speed, high-lift conditions are discussed, as well as attachment line contamination/transition concerns. This computational NLF design effort is a part of an ongoing cooperative agreement between NASA and JAXA researchers.
\end{abstract}

\section{Nomenclature}

$\begin{array}{ll}b & =\text { Span } \\ \text { beta } & =\text { Spanwise wave number } \\ c & =\text { Local chord } \\ C_{D} & =\text { Drag coefficient } \\ C_{L} & =\text { Lift coefficient } \\ C m & =\text { Pitching moment coefficient } \\ C_{p} & =\text { Surface pressure coefficient } \\ \operatorname{Re}_{c} & =\text { Reynolds number based on local wing chord } \\ \operatorname{Re}_{T} & =\text { Reynolds number based on streamwise transition location } \\ \operatorname{Re}_{\theta} & =\text { Reynolds number based on boundary layer momentum thickness } \\ (r / c)_{L E} & =\text { Leading-edge radius normalized by local chord } \\ S & =\text { Semispan, b/2 } \\ (t / c)_{\max } & =\text { Maximum thickness-to-chord ratio } \\ x / c & =\text { Streamwise location expressed as a fraction of local wing chord } \\ (x / c)_{T} & =\text { Streamwise location of transition expressed as a fraction of local wing chord } \\ x 1, x 2, x 3 & =\text { Design constraint location parameters } \\ y / s & =\text { Spanwise location as a fraction of wing span } \\ z / c & =\text { Vertical location expressed as a fraction of local wing chord }\end{array}$

${ }^{1}$ Research Aerospace Engineer, Configuration Aerodynamics Branch, NASA Langley Research Center M/S 499 Hampton, VA 23681, and AIAA Member.

${ }^{2}$ Senior Research Engineer, Configuration Aerodynamics Branch, NASA Langley Research Center M/S 499 Hampton, VA 23681, and AIAA Associate Fellow. 


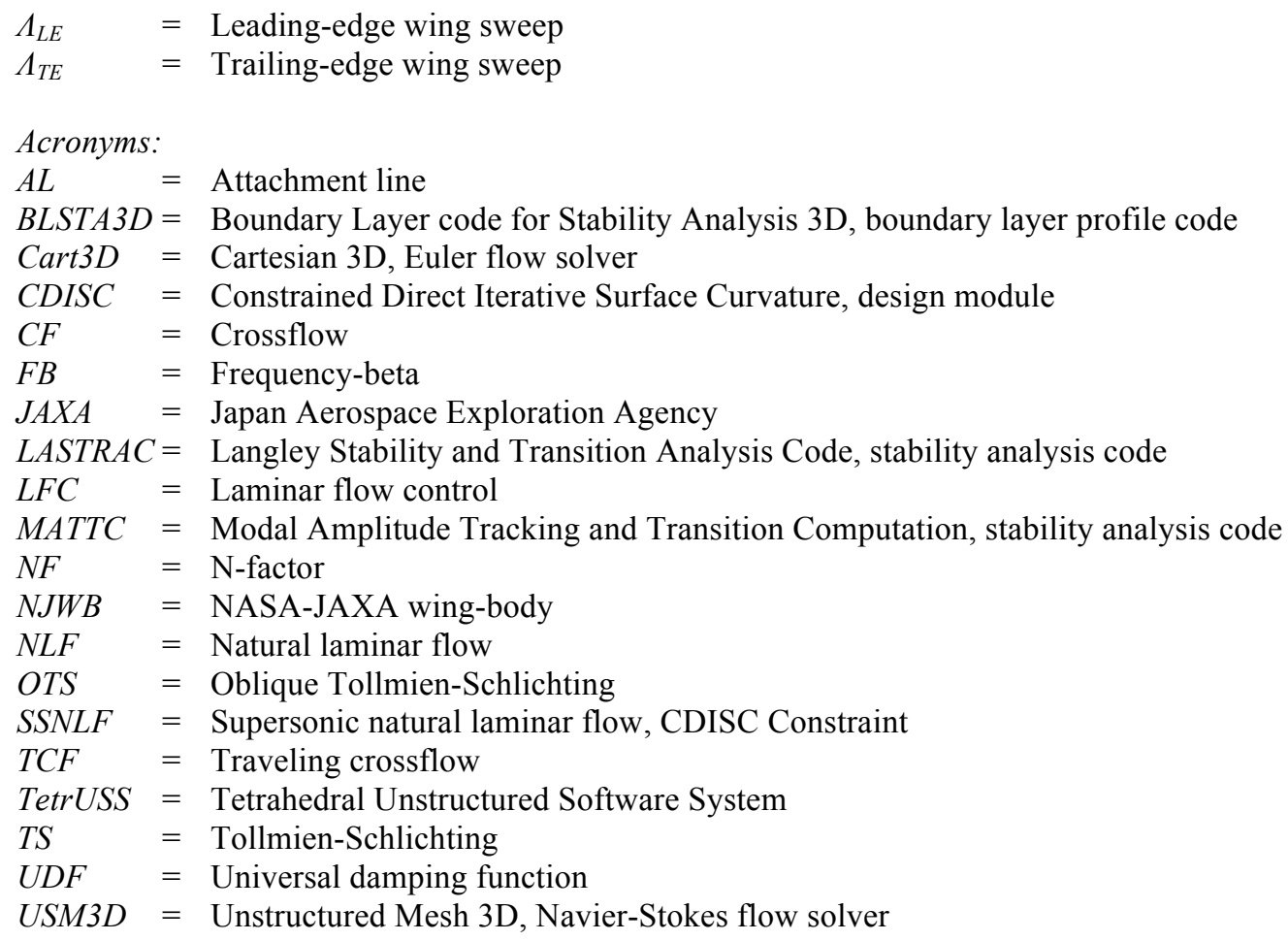

\section{Introduction}

N unrelenting push to decrease the environmental impact of aircraft drives research of drag-saving technology. - Current industry demands are also pushing for the return of supersonic commercial flight with faster, quieter, and cost-effective aircraft. Several private and public companies are pursuing efficient low-boom supersonic commercial transport designs to make these goals a reality. New research must be conducted to develop key technologies essential for the realization of efficient and quiet supersonic commercial flight. One such area of research is the practical application of laminar flow to supersonic transports. Presently, aircraft fly with a turbulent boundary layer over almost all surfaces. While a laminar boundary layer does have associated penalties, a turbulent boundary layer has significantly higher skin friction drag than a laminar boundary layer. As skin friction drag is a major source of a vehicle total drag, reducing the skin friction offers the potential of greatly reducing total drag. The aerodynamic performance benefit acquired from sustaining laminar flow can translate into reduced operating costs of an aircraft.

For supersonic flight, there are three types of boundary layer transition mechanisms that need to be addressed in order to sustain laminar flow: attachment line (AL) contamination/transition, Görtler vortices, and modal disturbances ${ }^{1}$. The first of these, AL contamination/transition, results in the loss of all possible laminar flow over the wing if not properly addressed. AL contamination is when the fuselage turbulent boundary layer runs out onto the leading edge of the wing. There are two solutions to avoid this phenomenon. A geometry feature, such as a Gaster bump $^{2}$, works by diverting the fuselage turbulent boundary layer away from the wing leading edge. The second solution is applying Poll's criterion ${ }^{3}$ to the wing leading-edge radius. This requirement, centered on limiting the Reynolds number based on boundary layer momentum thickness $\left(\mathrm{Re}_{\theta}\right)$ to less than 100 , leads to relatively sharp airfoil leading-edge geometries. AL transition occurs when the point of flow attachment has too large a radius to avoid disturbance transition along the AL. To prevent AL transition, a $\mathrm{Re}_{\theta} \leq 235$ must be maintained along the span of the wing at the attachment point. Both AL contamination and transition must be addressed to allow laminar flow over the wing. The second boundary layer transition mechanism, Görtler vortices, are counter-rotating vortices that induce transition and are caused by concavity of the surface. In this application, a surface curvature constraint is implemented to eradicate any concave regions in the desired laminar flow sections. The final boundary layer transition mechanism to address is modal disturbances. Modal disturbances can be further categorized into two distinct types: crossflow (CF) waves and Tollmien-Schlichting (TS) waves. At supersonic speeds, variations of these two types, known as traveling crossflow (TCF) and oblique Tollmien-Schlichting (OTS) waves, can also be significant. As the stability analysis for both the TCF and OTS variations involve non-zero frequency and spanwise 
wave number (beta) values, they will be collectively referred to as frequency-beta (FB) waves. Additional detail about the present definitions of CF, TS, and FB waves in relation to the boundary layer stability analyses can be found in Section II. Method, B. Transition Prediction Modules. These modal disturbance instabilities are associated with boundary layer eigenmodes and each responds differently to pressure gradients (stabilizing or destabilizing). The instabilities may propagate and amplify, ultimately leading to transition in different regions over the wing. The present research focuses primarily on damping modal disturbances, although the two other transition mechanisms are also addressed.

In supersonic applications, CF and TCF disturbances are the most dominant modes in the leading-edge region of wings. Historically, these disturbances are controlled through either limiting the leading-edge sweep to less than 20 degrees or introducing a flow control system. To reach supersonic flight conditions, the leading-edge sweep of a

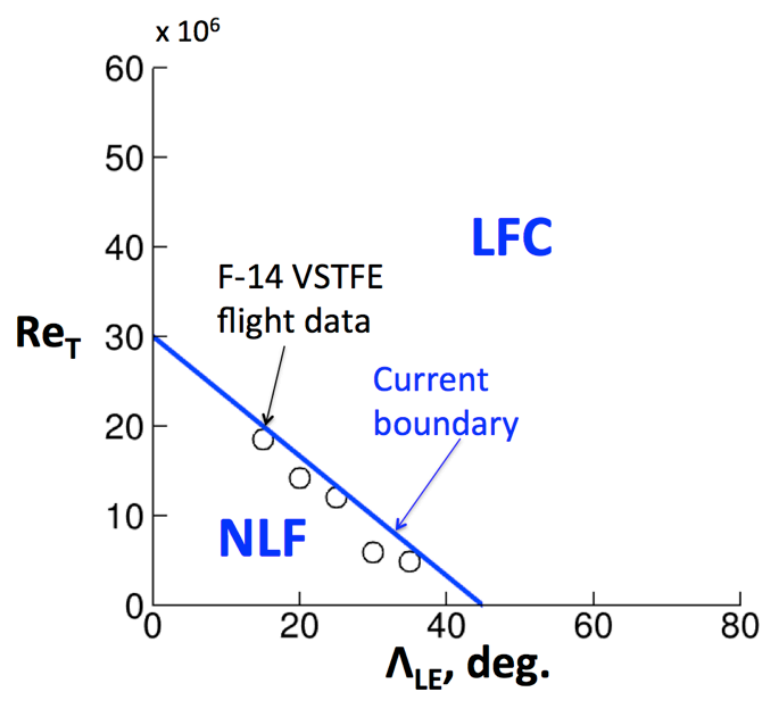

Figure 1. Current NLF-LFC boundary in terms of leading-edge sweep $\left(\Lambda_{\mathrm{LE}}\right)$ and transition Reynolds number $\left(\operatorname{Re}_{\mathrm{T}}\right)$. wing is typically much more than 20 degrees, although some aircraft such as the F-104 and Aerion's proposed supersonic business jet ${ }^{4}$ with natural laminar flow (NLF) have wings with fairly low leading-edge sweep. The application of NLF is limited by a combination of Reynolds number based on streamwise transition location $\left(\operatorname{Re}_{\mathrm{T}}\right)$ and the leading-edge sweep $\left(\Lambda_{\mathrm{LE}}\right)$. Presently, one of the highest published combinations of transition Reynolds number and leading-edge sweep in flight was on the F-14 VSTFE flight test ${ }^{5}$. This represents the boundary, shown in Figure 1, between aircraft with NLF and laminar flow control (LFC). An LFC system employs suction at the leading edge through a porous or slotted surface to damp the CF instability. While such systems have proven to successfully delay transition due to $\mathrm{CF}$, the added system complexity and weight have rendered this solution too costly for practical use in many situations. The major benefit of controlling transition due to $\mathrm{CF}$ waves with the present method is that it allows the vehicle to obtain the benefits of laminar flow (such as reduced skin friction drag), while permitting high leading-edge sweeps and removing the added cost associated with a complex suction system. The goal of this research is to push the current NLF boundary to higher combinations of transition Reynolds numbers and leading-edge sweep on supersonic commercial transports.

The NLF design technique described here stems from a collaborative effort between researchers at NASA and the Japan Aerospace Exploration Agency (JAXA) and was motivated by previous design efforts involving JAXA personnel $^{6,7}$. The method works by altering the pressure distribution to damp the dominant form of instability waves. The current paper describes an application of this NLF design method to a supersonic transport configuration referred to as the NASA/JAXA wing-body (NJWB). A companion paper entitled Natural Laminar Flow for Wings with Moderate Sweep reports the concurrent effort to apply a modified version of the new NLF design approach to transonic transports.

\section{Method}

The proposed NLF analysis and design method is comprised of a flow solver, grid manipulation tools, a design module, and transition prediction software, all of which are illustrated in the flow chart in Figure 2. The design and transition prediction loops in the figure are not performed at the same frequency in this application; the transition prediction loop (shown on the left) is done once a completed design is attained, in order to save computational time and resources.

In general, boundary layer profile information is needed for the stability analysis methods used to predict where the laminar boundary layer will transition to turbulent flow. This information can be extracted directly from a Navier-Stokes flow solution, but it requires larger grids to obtain the needed resolution of the profiles and the extraction process can be time consuming and difficult. In this study, an external boundary layer code is used that only requires streamwise pressure distributions to determine the edge conditions for the profiles. This approach thus is compatible with both inviscid or viscous flow solvers, and reduces the computational resources required for Navier-Stokes codes. As the Cart3 $\mathrm{D}^{8}$ code used in this study is an inviscid Euler flow solver, no transition location 


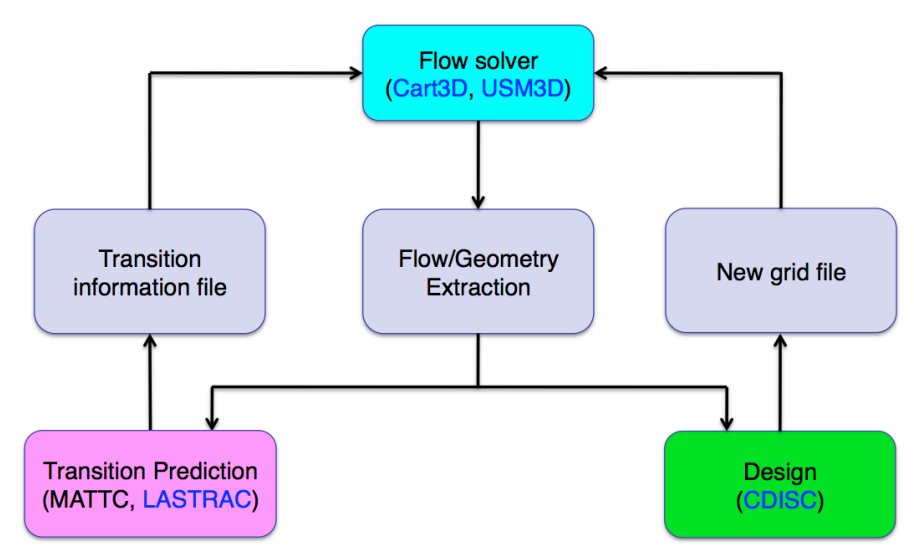

Figure 2. Flow chart of the NLF analysis and design framework.

feedback is needed for its use. However, the

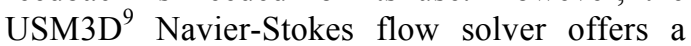
forced laminarization option that requires this feedback to that flow solver.

The following subsections offer details on the tools employed for the flow solvers, transition prediction modules, and design modules.

\section{A. Flow Solvers}

Two flow solvers were used in this NLF design approach application: Cart3D and USM3D. Cart3D (Cartesian 3D) is a highfidelity inviscid analysis package that uses geometry entered in the form of surface triangulations ${ }^{8}$. An inviscid flow solver inherently has no boundary layer information, which is vital to investigating the effects of supporting laminar flow. As mentioned above, however, an external boundary layer solver will be used to generate the profile information needed for stability analyses based on wing pressure distributions. Previous experience has suggested that boundary layer effects on wing pressures are small for supersonic configurations at their cruise design condition, so the use of an Euler method should provide reasonable accuracy while reducing computational resources. The validity of this assumption is evaluated in Section IV. Results, B. NLF Design using USM3D. The inviscid flow solver has grid sequencing and restart capability, which were used to reduce design time. Another reason for utilizing Cart3D is the example configuration presented in this paper was originally designed with a Cart3D-compatible grid. A prescribed leading-edge grid refinement was conducted prior to design to further resolve this crucial region of the wing. Upon developing the NLF design method with the quicker inviscid flow solver Cart3D, a method for converting Cart3D grids into Navier-Stokes flow solver compatible grids was established.

The Navier-Stokes flow solver chosen for this application was USM3D (Unstructured Mesh 3D), which is a part of the Tetrahedral Unstructured Software System (TetrUSS) ${ }^{9}$. USM3D is a cell-centered, finite-volume NavierStokes flow solver that uses Roe flux-difference splitting ${ }^{10}$ to compute inviscid flux quantities across the faces of tetrahedral cells. A grid for USM3D is generated from a Cart3D tri file using grid-generating software in TetrUSS. A triangular surface mesh is generated, after which viscous grid cells are built using an advancing layers method, followed by the remaining inviscid portion using an advancing front method. These unstructured grids had a $\mathrm{y}^{+}=$ 0.5 for the first cell center off of the surface. As the NLF design methodology developed, new features led to further localized grid improvement, such as refinement of the wing leading edge and additional viscous layers. The flow solutions were obtained using the Spalart-Allmaras turbulence model in regions of turbulent flow. The forced laminarization feature in USM3D was employed for this application. This allows the appropriate representation of laminar flow features, such as reduced skin friction and boundary layer thickness, which is essential to evaluating the potential benefit of an NLF design. While USM3D does offer the ability to extract boundary layer profiles directly from the flow solution, for consistency with the initial design analyses from Cart3D, the external boundary layer solver is also used with the USM3D flow solutions to determine boundary layer state. USM3D is also used to validate the use of an inviscid analysis package, Cart3D, as the flow solver for the initial design stage.

\section{B. Transition Prediction Modules}

As seen in the flow chart above in Figure 2, two transition prediction modules were considered for this application, MATTC and LASTRAC. The Modal Amplitude Tracking and Transition Computation (MATTC) code $^{11}$ is an empirical method that computes the $\mathrm{n}$-factor (NF) growth curves directly from the pressure distributions at the wing design stations, and thus does not require a boundary layer extractor or solver. However, MATTC, at this time, is limited to subsonic free-stream flows, so the Langley Stability and Transition Analysis Code (LASTRAC) ${ }^{12}$ was selected as the boundary layer stability analysis tool. LASTRAC is a collection of methods ranging in fidelity from linear stability theory to linear/nonlinear parabolized stability equations (PSE) and secondary instability theory and is applicable in all speed regimes. The n-factor method using linear stability theory within LASTRAC is used to determine transition location in this example. BLSTA $3 \mathrm{D}^{13}$ is used as the boundary layer solver due to its compatibility with LASTRAC and its ability to model 3D (conical) flows. This boundary layer solver is used with both Cart3D and USM3D flow solutions. 
Transition prediction remains a nontrivial task for engineers and, while both LASTRAC and BLSTA3D prove to be useful tools, careful tailoring of the codes' inputs/outputs was necessary for practical design work. Automation to incorporate these tools into the present work is vital to streamlining the design process. In the automated process, BLSTA3D produces the velocity and temperature profiles information, and then LASTRAC runs linear stability theory on a set of 20 frequency/beta pairs scaled up from input values. Checks have been incorporated into the automation to ensure a reasonable set of frequency/beta pairs were used for each boundary layer profile. Figure 3 shows a sample suite of frequency and beta values for what this paper defines as CF, TS, and FB. The frequency-beta combinations that are known to be OTS and TCF waves are highlighted in the figure; however, the forthcoming NF plots will not specifically designate between OTS and TCF, but instead will refer to the analysis as FB.

The automated transition prediction process enables

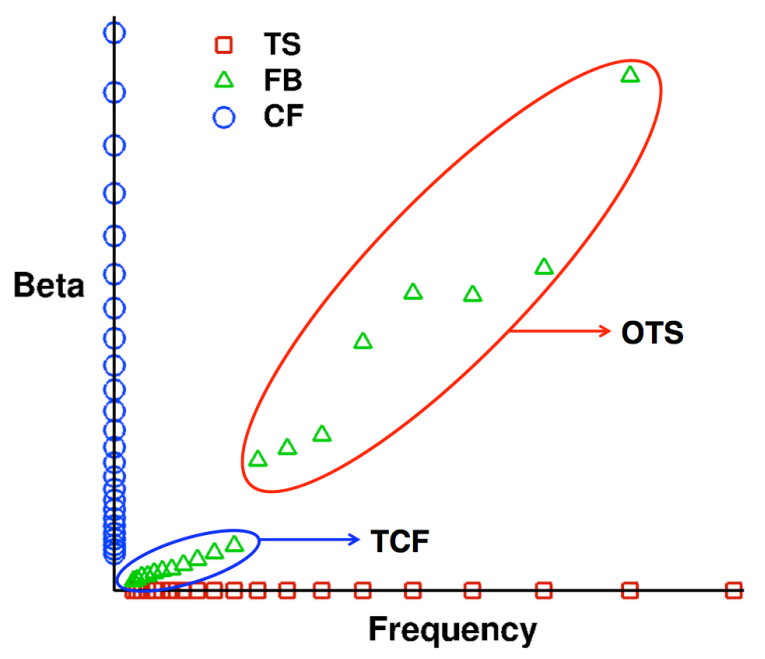

Figure 3. Example distribution of frequency-beta combinations for TS, FB, and CF. rapid boundary layer stability analysis for extracted streamwise pressures. This capability is used to evaluate the success of altering a supersonic configuration to support NLF using the design approach presented in the following section.

\section{Design Module}

The design tool selected for this research effort is the Constrained Direct Iterative Surface Curvature (CDISC) ${ }^{14}$ code, a knowledge-based design method compatible with a variety of 2D and 3D flow solvers. The code has been coupled with both Cart3D and USM3D flow solvers and their respective grids. CDISC uses specified flow/geometry relations, developed from analytical or empirical studies, to compute geometry changes based on the difference between current and target flow quantities. This technique greatly reduces design time by eliminating the need to compute sensitivity derivatives, allowing the design to converge in parallel with the flow solution, and using flow constraints to automatically develop the target pressure distribution from the current values of flow qualities such as pressure or skin-friction coefficients. CDISC flow constraints allow designing to common engineering variables, such as: span load, section lift and/or pitching moment coefficients, and shock strength. Similarly, geometry constraints are available to address requirements from other disciplines such as structures and manufacturing, including: thickness, curvature, volume, and leading-edge radius. For the NLF design effort presented here, some existing CDISC constraints were modified and a new flow constraint SSNLF (Supersonic Natural Laminar Flow) was developed. The SSNLF constraint is described in detail in the following section.

\section{Design Approach}

The present design approach that aims to achieve NLF on supersonic transport wings has been focused on answering the following three questions:

1) Can a target pressure distribution be defined that will support a significant region of laminar flow and meet other flow constraints (lift, pitching moment, shock strength, etc.)?

2) Can a geometry be designed to match the target pressures with sufficient accuracy to largely obtain the target laminar flow region while matching geometry constraints (thickness, leading-edge radius, twist, etc.)?

3) Does the attachment line for the design fall within the bounds defined by Poll's criteria ${ }^{3}$ to avoid attachment line contamination/transition?

This paper presents the current status of an NLF design effort to answer the above questions. If these aerodynamic-based questions can be successfully addressed, concerns relating to the practicality of such a wing still exist. Namely, issues such as cost of manufacturing and maintaining the finish quality of the wing during operation would need to be addressed to make NLF supersonic wings viable. The current effort is focused on answering the aerodynamic-based questions and does not address practicality concerns. This section discusses the design approach created within CDISC to determine NLF target pressures for a given configuration. 
In the CDISC design process, several existing geometry constraints were used to meet thickness, curvature, and leading-edge radius requirements, although the radius constraint did require modifications to handle the extremely small values needed to meet the AL contamination limit. A new flow constraint was developed to define a target pressure distribution that will damp the modal (TS, CF, and FB) disturbances as mentioned above. The approach used in the new constraint is discussed below.

The target pressure distribution is divided into 4 regions as shown in Figure 4 , where $\mathrm{C}_{\mathrm{P}}$ is the pressure coefficient and $\mathrm{x} / \mathrm{c}$ is the streamwise location expressed as a fraction of local wing chord. To limit the growth of $\mathrm{CF}$ and FB peaks near the leading edge, a rapid acceleration is prescribed from the attachment line to $\mathrm{x} 1$, followed by a short region of zero pressure gradient ending at $\mathrm{x} 2$. The location of $\mathrm{x} 1$ is obtained from a formula relating that location to peak n-factor (NF) level as a function of Reynolds number and wing sweep. This formula is plotted in Figure 5 as lines, along with the symbols for LASTRAC analyses of different values of Reynolds number and wing sweep.

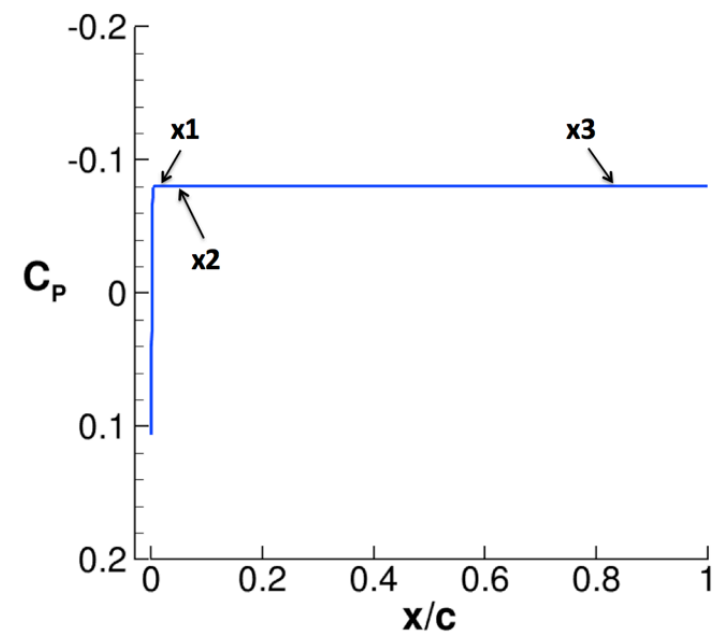

Figure 4. NLF target upper surface $C_{P}$ layout showing relative locations of $x 1, x 2$, and $x 3$.

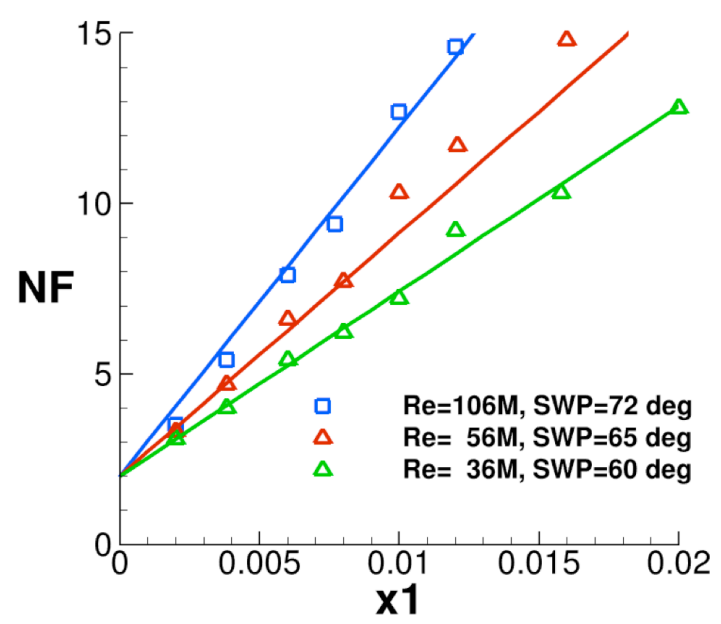

Figure 5. Peak NF versus $x 1$ location for Re and sweep combinations.

Figure 6 shows LASTRAC analysis results for the swept flat pressure distribution shown in Figure 4, where x1 and the attachment line $\mathrm{C}_{\mathrm{P}}$ correspond to a Mach number of 1.6, a chord Reynolds number of 106 million, a leadingedge sweep angle of 71.6 degrees, and a critical NF of 9 (represented by the horizontal black line). The leading-edge peak is slightly higher for FB (Figure 6b) than for CF (Figure 6c) and is consistent with the desired margin of 2-3 below the critical NF level. For FB, the critical level is reached, indicating transition from laminar to turbulent flow, slightly further downstream of the leading-edge peak. TS also crosses the critical level, but occurs slightly downstream of the FB transition $\mathrm{x} / \mathrm{c}$. 


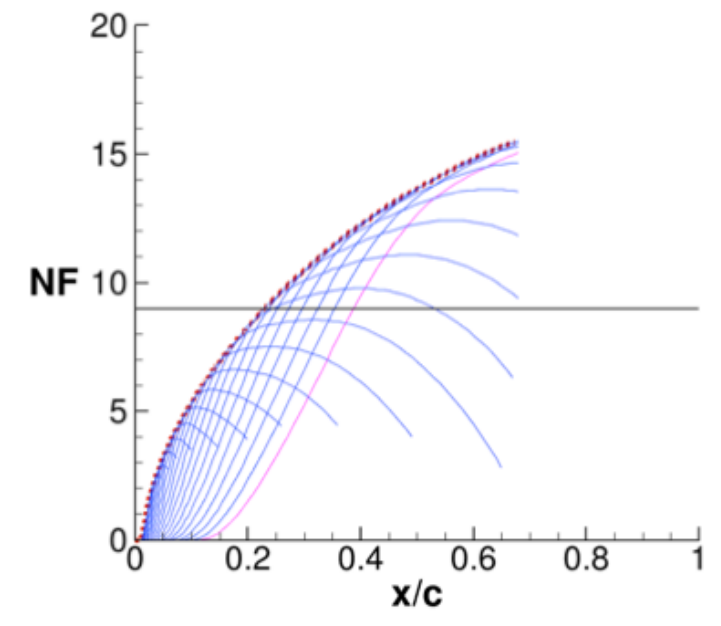

a) TS.

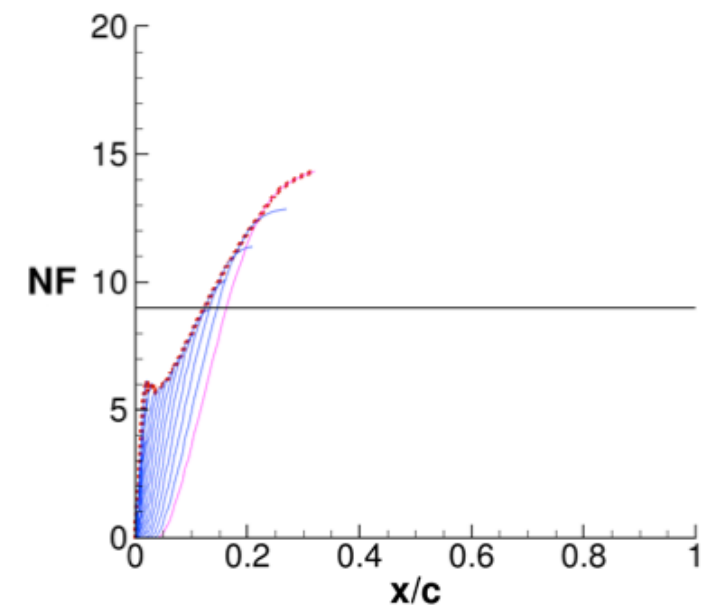

b) FB.

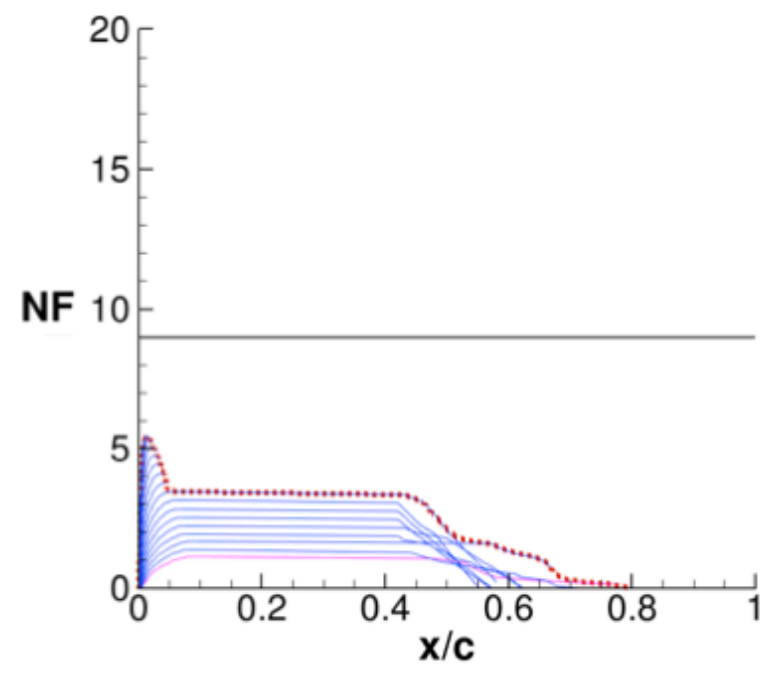

c) $\mathrm{CF}$.

Figure 6. LASTRAC analysis for a swept flat plate case at $\mathrm{Mach}=1.6, \operatorname{Re}_{\mathrm{c}}=106 \mathrm{M}, \Lambda_{\mathrm{LE}}=71.6 \mathrm{deg}$.

In order to push back the FB transition location, a pressure gradient function, referred to as the universal damping function (UDF), is added between $\mathrm{x} 2$ and $\mathrm{x} 3$ (see Figure 4). This function is so-named because, with the specification of a single parameter, the UDF can essentially flatten the TS growth at a desired NF level (Figure 7a) or, if applied with the opposite sign, can stop mid-chord FB growth (Figure 7b). Unfortunately, the UDF sign change implies that shifting the FB transition location aft will increase the TS growth rate; thus the optimum extent of laminar flow will require a balance between these two modes. 


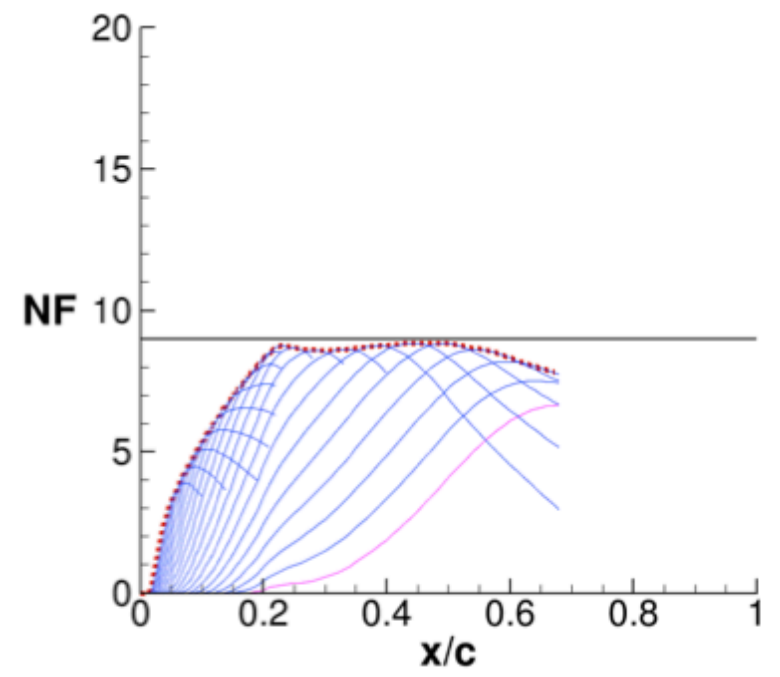

a) TS.

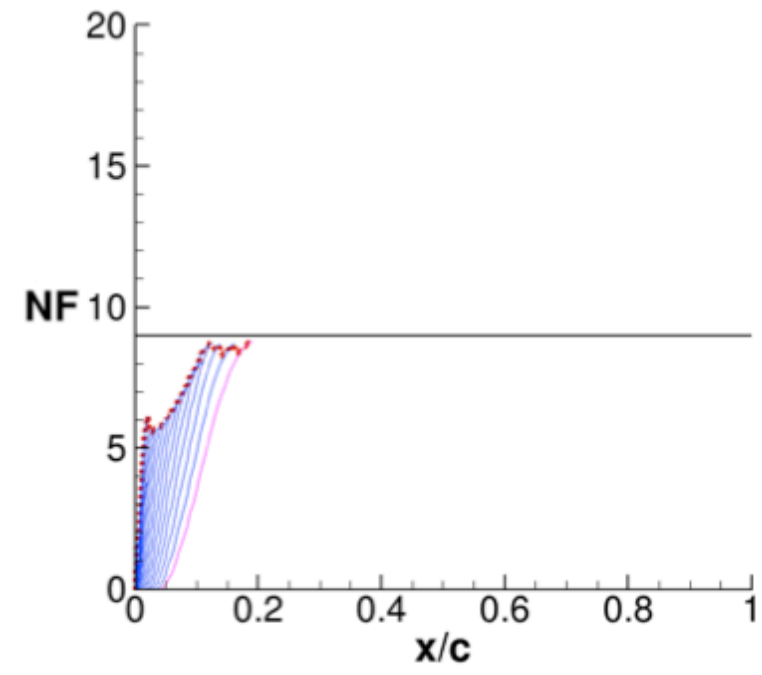

b) FB.

Figure 7. Applications of UDF to stop n-factor growth on the swept flat plate case. Left shows effects of the UDF used to damp TS, right shows effects of the UDF used to damp FB.

The NF envelope curves from Figure 6 are plotted in Figure 8a to show the spread between the FB and TS curves for the original flat target pressures. Figure $8 \mathrm{~b}$ shows the same analysis for a target distribution with a UDF segment added based on the formula in the CDISC NLF constraint. The two curves now come close to crossing the critical level at the same chordwise location, giving a near-optimum amount of laminar flow.

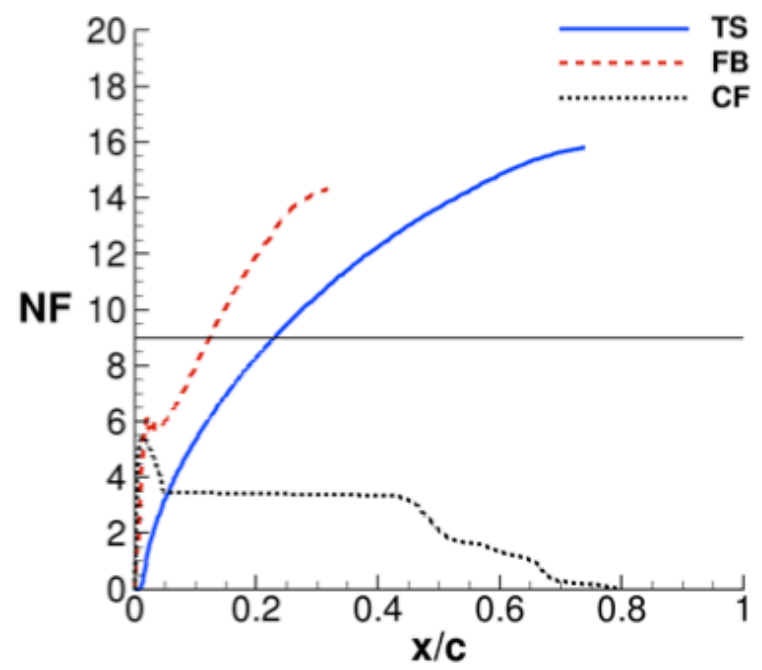

a) Unaltered.

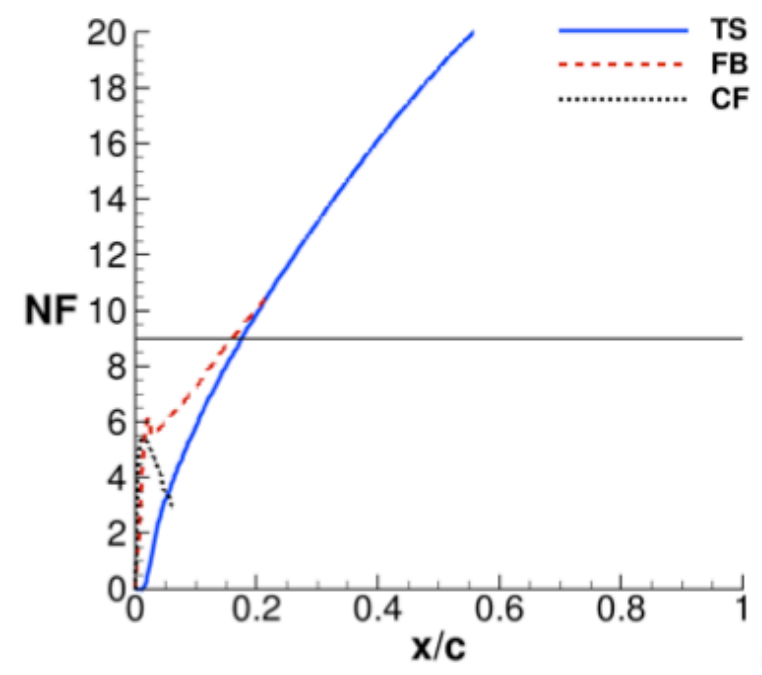

b) Balanced UDF segment.

Figure 8. Effect on transition location of the UDF balanced for both TS and FB on the swept flat plate case.

Using this approach, CDISC will estimate an $\mathrm{x} 3$ location to provide the maximum extent of laminar flow on a configuration for a specified critical NF and only apply the UDF value ahead of this location. Aft of that location, the target pressures are blended back to the baseline $C_{P}$ values to minimize the inviscid drag penalty caused by the changes to the original turbulent flow optimization. In the next section, this approach will be demonstrated on a more realistic supersonic configuration. 


\section{Results}

The aforementioned NLF design method is applied to a supersonic configuration to demonstrate the technique. The example configuration, referred to as the NASA-JAXA Wing Body (NJWB), is a generic Concorde-size supersonic transport designed using Cart3D adjoint-based optimization. The NJWB design objective was to provide good aerodynamic performance assuming turbulent flow and a wing planform and fuselage compatible with low sonic boom requirements.

NLF was only attempted on the wing upper surface in this application. This was to reflect a practical application where wing features leading to surface gaps or steps, such as a Krueger flap or access panels, would cause boundary layer transition along the lower surface. In addition, this application limited the extent of laminar flow to $\mathrm{x} / \mathrm{c}=0.85$ to account for the loss in laminar flow over a flap or aileron.

The vehicle was designed to cruise at Mach 1.6 and 3 degrees angle of attack with a unit Reynolds number of 1.9 million per foot and a design lift coefficient $\left(\mathrm{C}_{\mathrm{L}}\right)$ of 0.1 . The

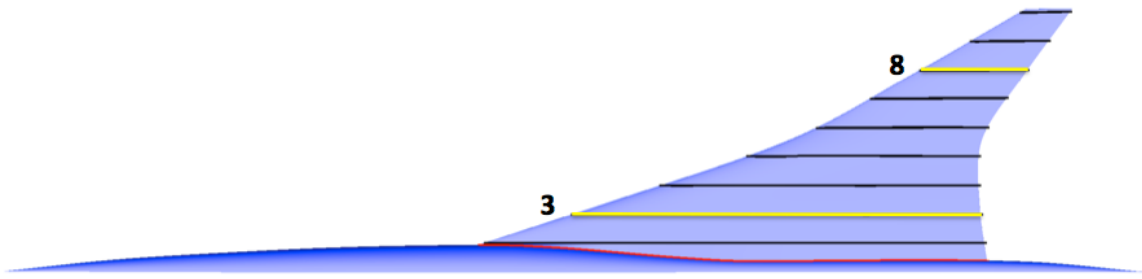

Figure 9. Design stations on NJWB configuration. Stations 3 and 8 (highlighted in yellow) will be used for presenting results.

NJWB configuration has 10 streamwise stations on the wing used for CDISC design. For brevity, two stations were selected to demonstrate the NJWB application of the NLF design and analysis procedure. To represent the general characteristics and differences of the inboard and outboard sections, Stations 3 and 8 were chosen, shown as yellow lines on Figure 9. Station 3, located at a semispan location (y/s) of 0.22 , has a Reynolds number based on local chord length $\left(\mathrm{Re}_{\mathrm{c}}\right)$ of 135.8 million, a leading-edge sweep $\left(\Lambda_{\mathrm{LE}}\right)$ of 71.9 degrees, and a trailing edge sweep $\left(\Lambda_{\mathrm{TE}}\right)$ of 6.7 degrees. Station 8, located at $\mathrm{y} / \mathrm{s}$ of 0.78 , has an $\operatorname{Re}_{\mathrm{c}}$ of 35.6 million, a $\Lambda_{\mathrm{LE}}$ of 60.2 degrees, and a $\Lambda_{\mathrm{TE}}$ of 36.3 degrees.

\section{A. NLF Design using Cart3D}

In an effort to reduce design time, the initial design of the NJWB configuration was performed using the inviscid Euler code, Cart3D, and is presented in this subsection. As mentioned in Section II. Method, A. Flow Solvers, an external boundary layer profile solver is used to characterize the boundary layer state based on the pressure distributions obtained from the flow solver. For the supersonic design conditions in the application, it is assumed that viscous effects do not significantly affect the wing pressures at the design stations. In order to evaluate the validity of this assumption, the final inviscid Cart3D design was transferred to a Navier-Stokes flow solver, USM3D, and reevaluated, the results of which will be described in the following subsection.

Pressure distribution modifications to the NJWB baseline that support NLF were applied using the CDISC SSNLF flow constraint. Figures 10 and 11 show the pressure distributions and airfoil geometries (with changes in local twist removed for visualization purposes) of Stations 3 and 8, respectively. The target and design pressures are nearly identical, indicating that a geometry exists that has the desired pressure distribution. 


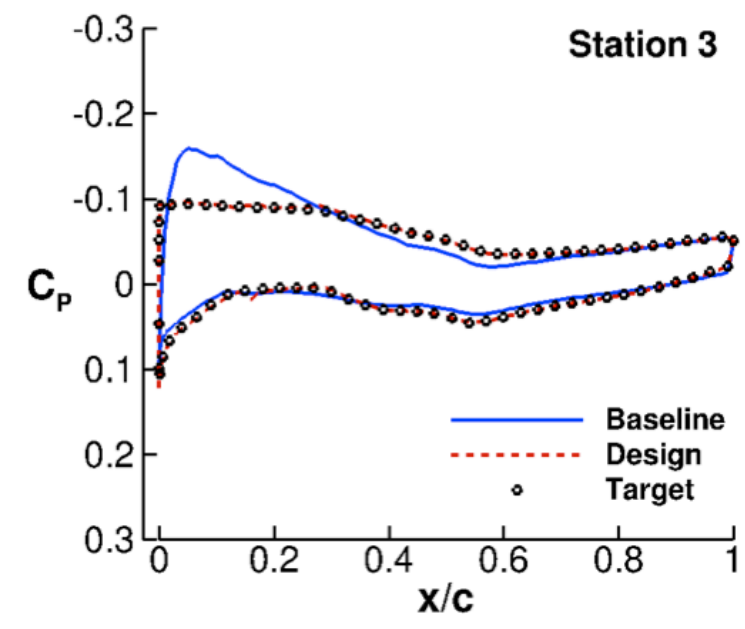

a) Pressure distributions.

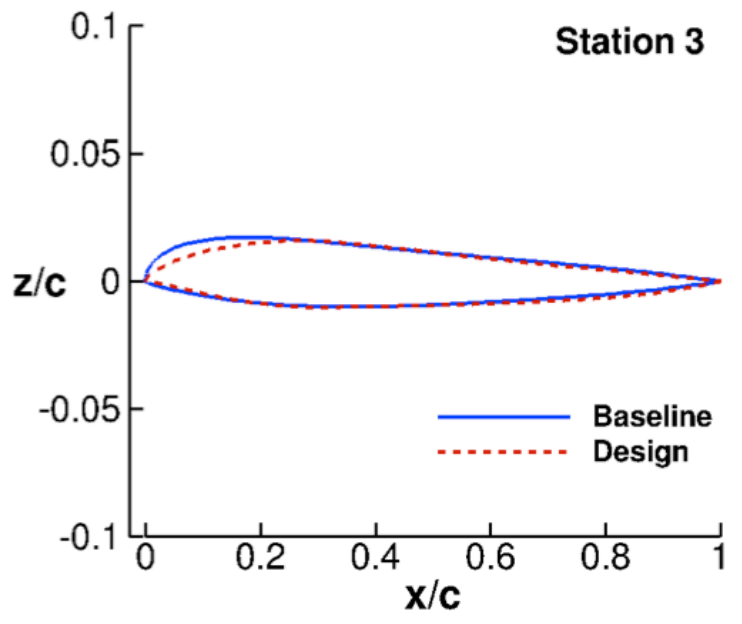

b) Airfoil geometries.

Figure 10. Results from Cart3D at Station 3. Left shows pressure distributions comparing Baseline, Design, and Target pressures. Right shows airfoil geometry changes between Baseline and Design with local twist removed.

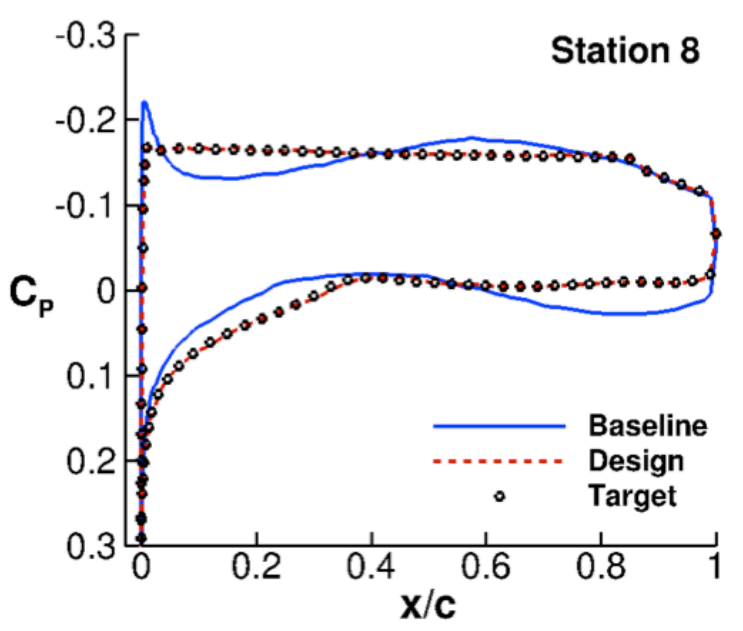

a) Pressure distributions.

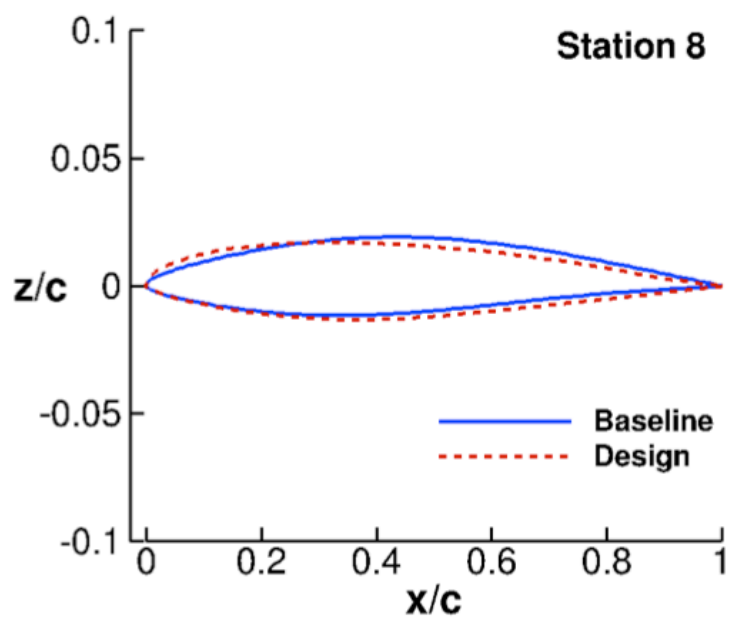

b) Airfoil geometries.

Figure 11. Results from Cart3D at Station 8. Left shows pressure distributions comparing Baseline, Design, and Target pressures. Right shows airfoil geometry changes between Baseline and Design with local twist removed.

At the leading edge, there is a short region of rapid flow acceleration followed by a quick leveling off of the $C_{P}$ to control transition due to CF. This pressure alteration, along with the aforementioned leading-edge radius constraint, causes a sharp nose on the Design airfoils. This sharpening is more prominent inboard and relaxes significantly over the outboard portion of the wing. Upon addressing transition due to CF instabilities, TS and FB are damped simultaneously through a region with a very mild adverse $C_{P}$ gradient. The slope and length in this region are determined by the UDF parameter described above. The end of this region signifies transition from laminar to turbulent flow and leads to the pressures on the Design blending back into the Baseline distribution. It can be visually deduced from the $C_{P}$ plots above that the NLF airfoil should be able to support laminar flow up to approximately $25 \%$ and $85 \%$ chord on the upper surfaces of Stations 3 and 8 , respectively, with the airfoil alterations illustrated. This prediction will be evaluated using boundary layer stability analysis and transition prediction modules.

Upon altering the grid geometry and pressure distributions, a boundary layer stability analysis is performed to confirm the estimated extent of laminar flow achieved. Each station's pressure distribution is evaluated using the boundary layer profile solver, BLSTA3D, and stability analysis code, LASTRAC, both of which are described in 
Section II. Methods, B. Transition Prediction Module. The critical NF level used with the LASTRAC results to identify transition location is set to 13 (represented by the solid black horizontal line on the NF vs. $\mathrm{x} / \mathrm{c}$ plots). This value is slightly less than the value of 14 reported by Belisle ${ }^{15}$ for a highly-polished leading edge and may be considered as somewhat of an upper bound for designs. A CF analysis (not shown) indicated that the pressure alterations made near the leading edge, namely the rapid acceleration and flattening out, successfully damped CF instability waves. This leaves the two dominant modes of transition for this configuration as TS and FB, so, while $\mathrm{CF}$ analysis was still performed and checked during the transition prediction process, only the results of TS and FB will be shown. As previously mentioned, the TS and FB transition mechanisms have opposite responses to pressure gradients, i.e., when one is damped the other grows, and the UDF parameter works to balance these two modes. This suggests that to support the largest extend of laminar flow, the $\mathrm{x} / \mathrm{c}$ locations where the NF curves first reach critical NF should be reasonably close for both the TS and FB analyses. The NLF Design configuration successfully demonstrated this balancing feature, seen in Figure 12 below. The stability analysis predicted Station 3 could sustain laminar flow up to $22 \%$ and $28 \%$ chord for transition due to TS and FB, respectively. The agreement between the $\mathrm{TS}$ and $\mathrm{FB} \mathrm{x} / \mathrm{c}$ transition locations suggests that the pressure distribution is fairly well optimized to provide the largest possible region of laminar flow for the spanwise location of this wing.

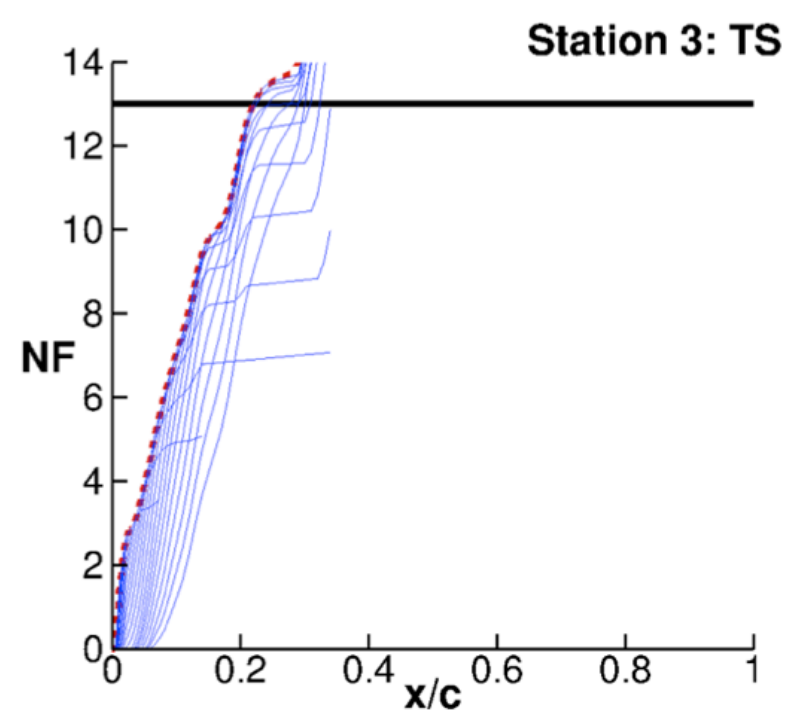

a) TS.

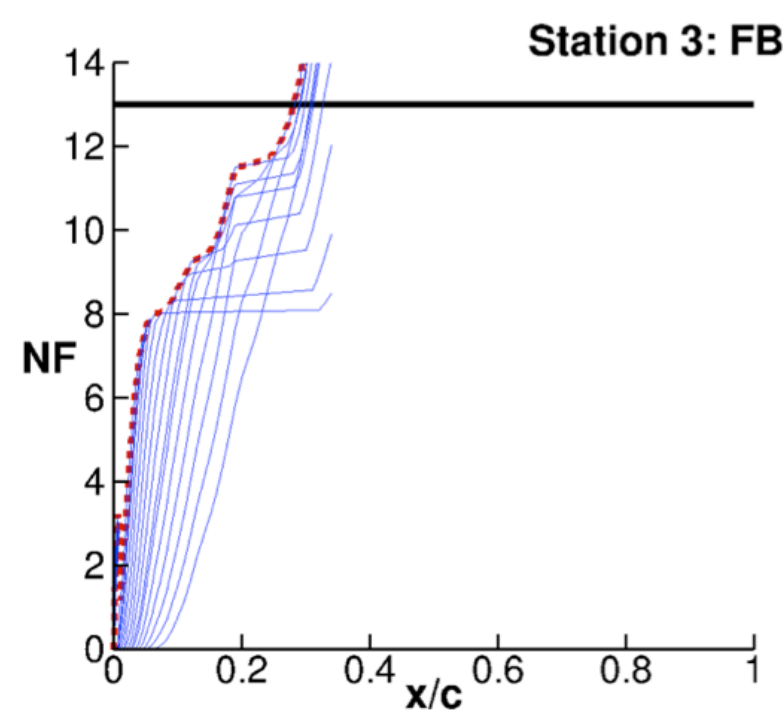

b) FB.

Figure 12. LASTRAC stability analysis of NLF Design pressures at Station 3.

For the cases where the critical NF value is never achieved with a given mode, a transition location estimator is employed. As noted above, visual inspection suggests that the laminar boundary layer would transition soon after the region of relatively flat $\mathrm{C}_{\mathrm{P}}$ level due to the strong adverse pressure gradients. With this knowledge, a pressure gradient limit was integrated into the boundary layer profile solver, BLSTA3D, which increases the robustness of the code. If the critical NF value is never realized, as is the case in Figure 13 below, the transition location is taken as the extent of the boundary layer solution. This transition location estimator is known to be slightly conservative, however, evaluating several pressure distributions confirms it as a reasonable approximation. 


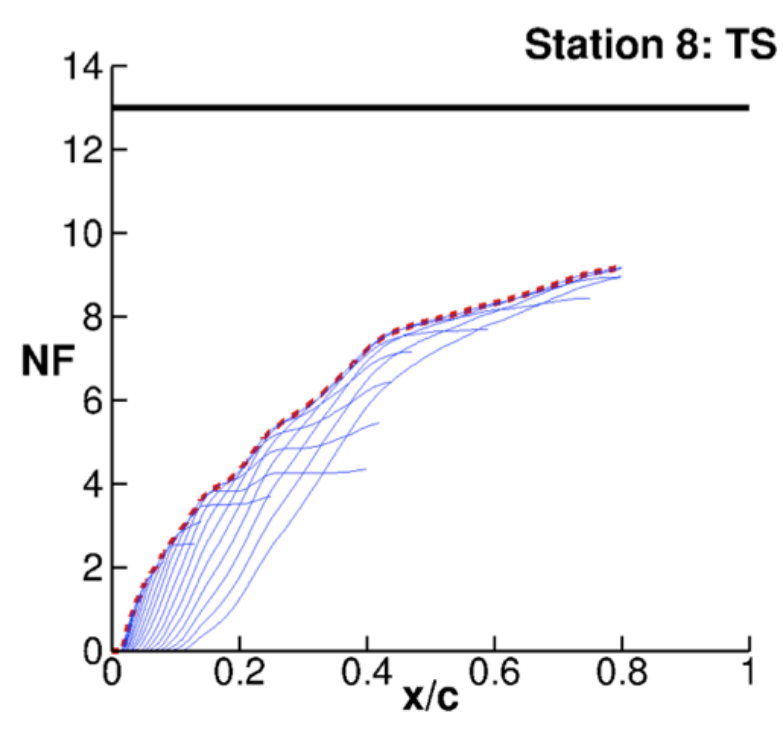

a) TS.

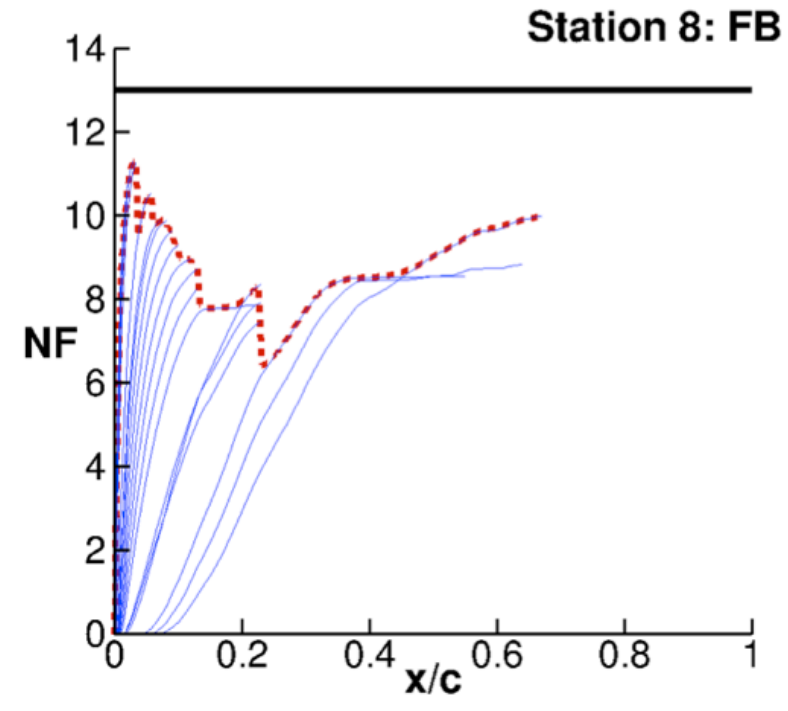

b) FB.

Figure 13. LASTRAC stability analysis of NLF Design pressures at Station 8.

Upon evaluating transition due to CF, TS, and FB modes at each station, a transition front can be determined. Spanwise transition location is determined to be the lowest transition $\mathrm{x} / \mathrm{c}$ from the three modes at each station. The results are interpolated between stations to produce the transition front shown on the planform view in Figure 14. This image shows both the transition front estimated from the target pressures and the final design pressures. The significant extend of laminar flow (approximately $44 \%$ of the wing upper surface area), as well as the similarity between the target and design pressures, suggest affirmative conclusions to questions 1 and 2 listed in Section III. Design Approach.

Successful alteration of the NJWB configuration to support NLF over the upper surface of the wing leads to changes in the aerodynamic performance of the vehicle. As Cart3D is an inviscid flow solver, a slip boundary condition is applied at the surface; thus, no skin friction can be generated. In order to estimate a skin friction value, an empirical method for predicting laminar and turbulent contributions to the skin friction drag was run. This technique approximates the surface area of the laminar region based on the computed transition locations. It then uses analytical skin friction equations for both laminar and turbulent flow to calculate the difference between a fully turbulent wing and the new partially laminar wing. For the NJWB NLF Design example, approximately $44 \%$ of the wing upper surface area is laminar. This laminar area accounts for an estimated 6.4 drag count savings over a fully turbulent boundary layer. Cart3D gave an inviscid drag penalty $\left(C_{D}\right.$ increase) of 3.4 counts from the NJWB Baseline to the NLF Design. Combining the two drag changes suggests that the net benefit in drag from sustaining a laminar boundary layer on this configuration

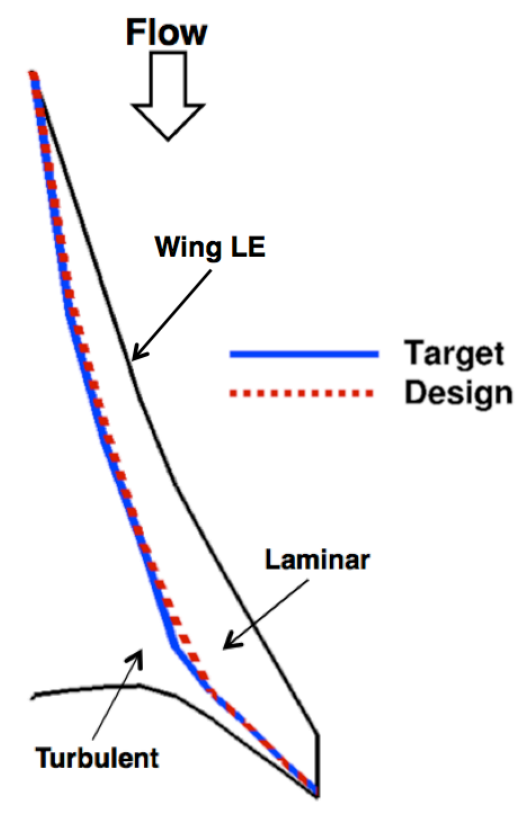
is 3 counts. It should be noted that this method did not account for any profile drag benefits accruing due to the thinner turbulent boundary layer at the wing trailing edge for the NLF Design. While this aerodynamic performance estimate is useful for design stage evaluation, a more complete analysis of the final NLF Design will be necessary to validate these findings. 


\section{B. NLF Design using USM3D}

To conduct a more complete evaluation of the NLF Design and to validate the independent skin friction drag calculator, the final Cart3D grid was converted to a USM3D-compatible grid to be analyzed with viscous effects. The grid conversion process led to slight differences in the Cart3D and USM3D geometries. CDISC design was conducted on the USM3D grid, applying several smoothing cycles as well as the same combination of geometry and flow constraints that produced the original Cart3D NLF Design. The final pressure distributions are compared in Figure 15 and show good agreement between the two flow solvers.

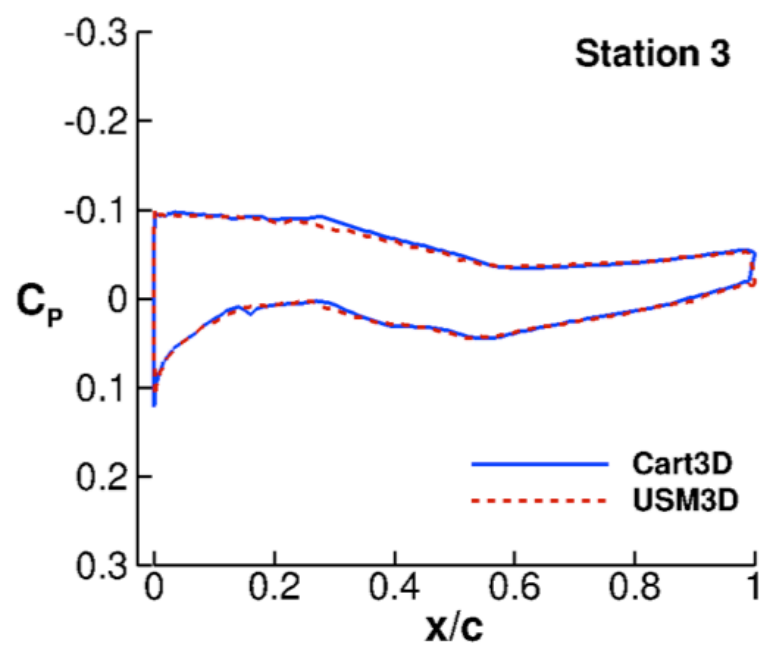

a) Station 3 .

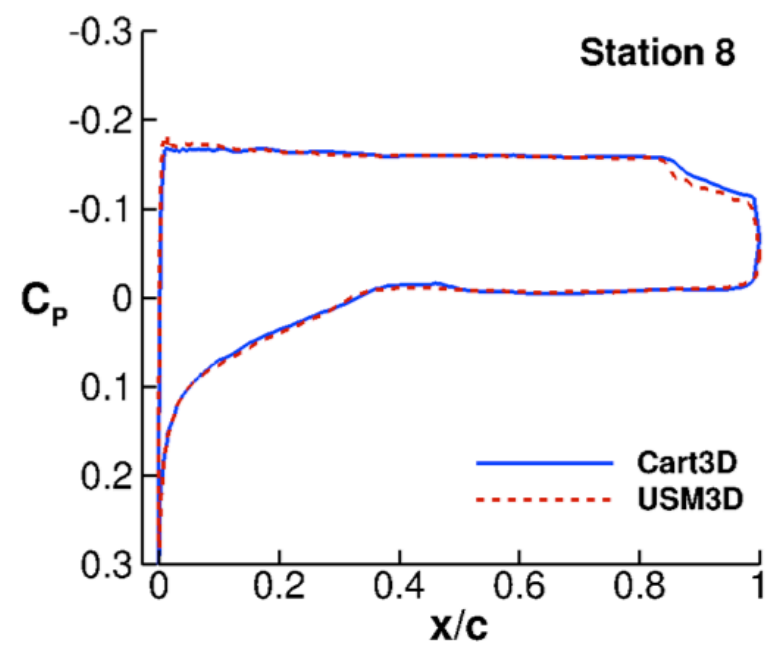

b) Station 8 .

Figure 15. Pressure distributions comparing the NLF Design from Cart3D and USM3D.

The transition fronts, compared in Figure 16, also illustrate good agreement, matching transition $\mathrm{x} / \mathrm{c}$ within an average of 3\% chord. The USM3D NLF Design sustained laminar flow over approximately $40 \%$ of the upper surface area. The similarity between the Cart3D and USM3D pressures and transition prediction results confirms the assumption that viscous effects are small relative to the NLF pressure architecture, validating the use of Cart3D as an initial design tool.

The USM3D analysis will be used as the final NLF Design for this application. Relevant transition parameters are shown in Table 1 for each station where design was performed. Several spanwise plots are shown in Figure 17 to further illustrate the geometry changes needed to acquire the desired NLF pressure distributions. 


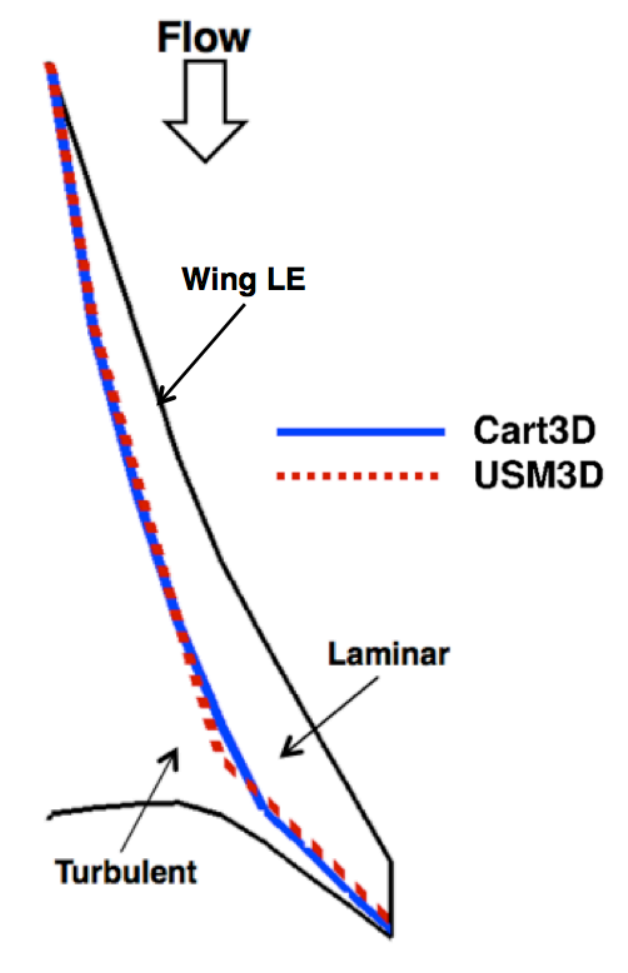

Figure 16. Cart3D and USM3D transition fronts showing laminar and turbulent flow regions on the planform of the wing.

Table 1. Transition parameters at each station used for design from USM3D

\begin{tabular}{|c|c|c|c|c|c|c|c|c|}
\hline Station & 3 & 4 & 5 & 6 & 7 & 8 & 9 & 10 \\
\hline $\mathbf{y} / \mathbf{s}$ & 0.22 & 0.33 & 0.45 & 0.55 & 0.66 & 0.78 & 0.89 & 1.00 \\
\hline chord (ft) & 71.5 & 55.6 & 40.4 & 29.7 & 23.7 & 18.7 & 13.6 & 8.8 \\
\hline $\mathbf{R e}_{\mathbf{c}}(\mathbf{M i l l i o n})$ & 135.8 & 105.7 & 76.7 & 56.5 & 45.0 & 35.6 & 25.9 & 16.7 \\
\hline $\boldsymbol{\Lambda}_{\mathbf{L E}}$ & $71.9^{\circ}$ & $71.6^{\circ}$ & $69.6^{\circ}$ & $64.8^{\circ}$ & $60.9^{\circ}$ & $60.1^{\circ}$ & $60.0^{\circ}$ & $59.8^{\circ}$ \\
\hline $\boldsymbol{\Lambda}_{\mathbf{T E}}$ & $-6.8^{\circ}$ & $-3.9^{\circ}$ & $6.9^{\circ}$ & $24.7^{\circ}$ & $34.8^{\circ}$ & $36.3^{\circ}$ & $36.6^{\circ}$ & $37.1^{\circ}$ \\
\hline$(\mathbf{x} / \mathbf{c})_{\mathbf{T}}$ & 0.23 & 0.34 & 0.46 & 0.60 & 0.83 & 0.83 & 0.90 & 0.90 \\
\hline $\mathbf{R e}_{\mathbf{T}}(\mathbf{M i l l i o n})$ & 30.6 & 36.2 & 35.0 & 33.9 & 37.8 & 29.7 & 23.3 & 15.0 \\
\hline
\end{tabular}




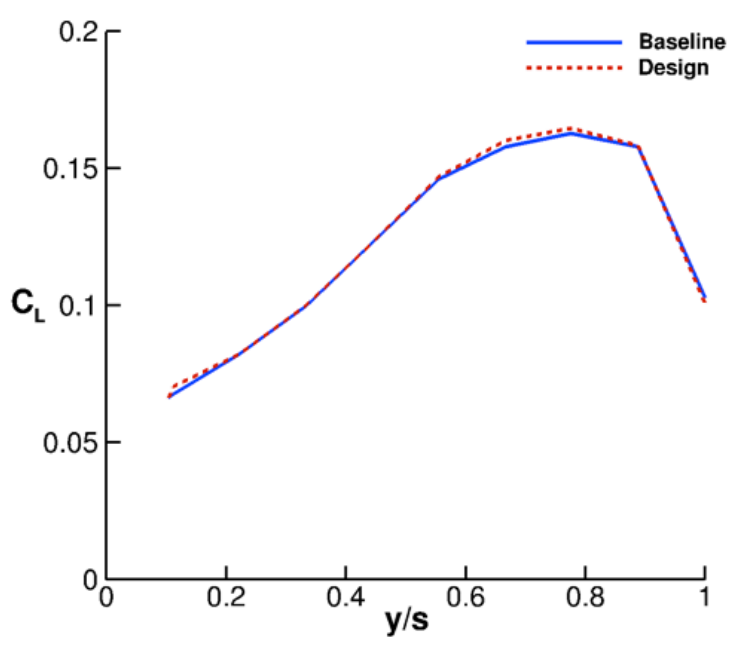

a) Lift coefficient.

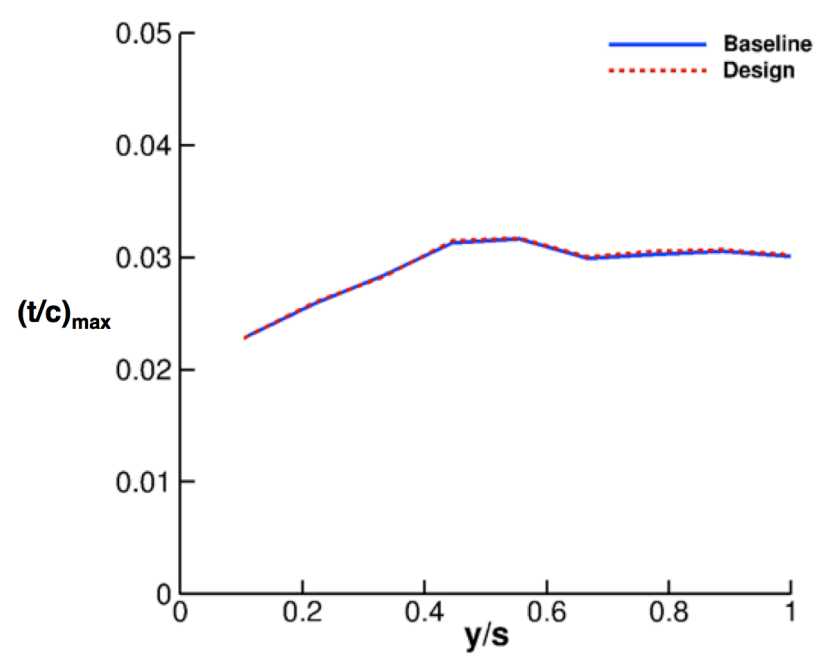

c) Maximum thickness-to-chord ratio.

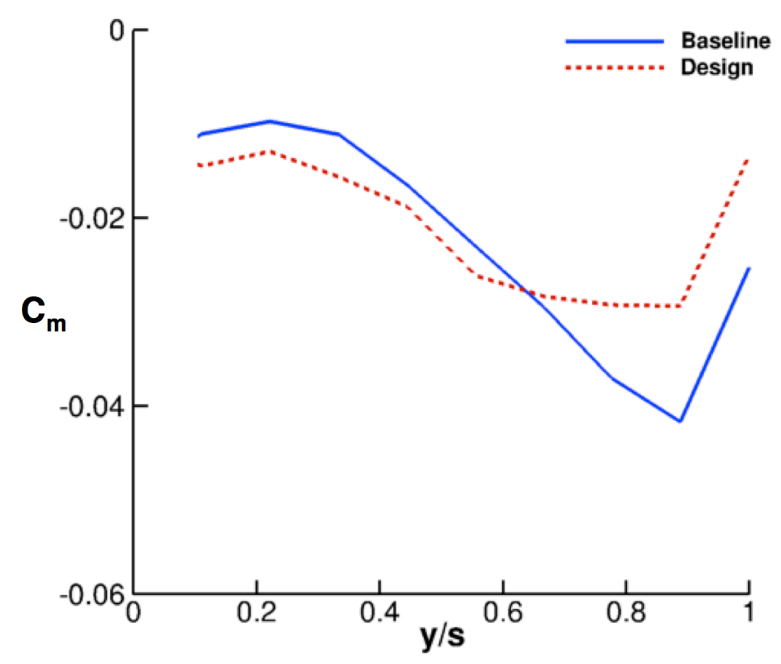

b) Pitching moment coefficient.

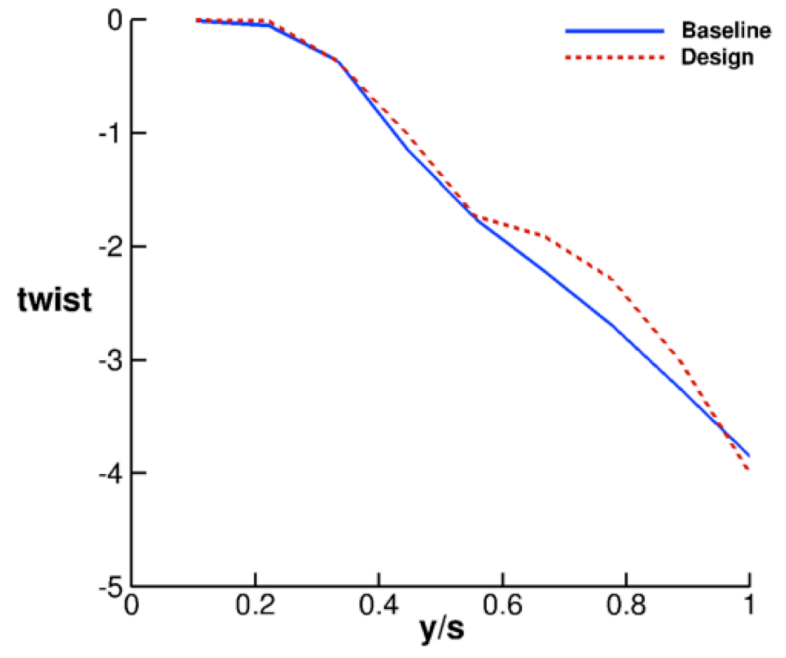

d) Twist in degrees.

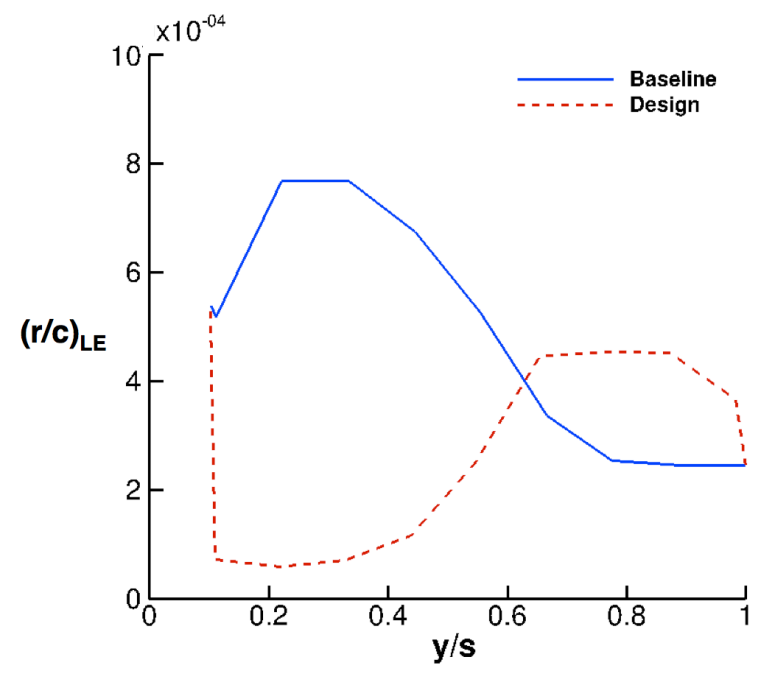

e) Leading-edge radius normalized by local chord.

Figure 17. Comparison of Baseline and Design spanwise characteristics from USM3D results. 
The section lift and maximum thickness ratio were held constant during the design process through constraints in CDISC. The spanwise pitching moment and twist saw some changes during the design, but maintained the overall characteristic of the baseline configuration. The total pitching moments between the two configurations were very similar, the design being slightly more negative than the baseline. The most altered spanwise distribution is the leading-edge radius normalized by local chord, where the NLF Design curve illustrates the effects of the leadingedge radius constraint (enforcing $\mathrm{Re}_{\theta} \leq 100$ ) implemented in the CDISC design. As mentioned previously, the smaller radii are primarily seen inboard, whereas the outboard stations of the wing experience an increase from the Baseline. Further discussion of the effectiveness of this leading-edge radius constraint on the attachment line contamination/transition is presented in a later subsection.

By utilizing the feature in USM3D that enforces laminar flow ahead of a designated transition front, a more accurate evaluation of the total drag savings the NLF Design offers can be obtained. Table 2 shows the drag components for the Baseline and NLF Design at the design cruise lift $\left(\mathrm{C}_{\mathrm{L}}=0.1\right)$. Additionally, this table reports the NLF Design geometry analyzed fully turbulent, which is representative of how the vehicle would perform if all laminar flow were lost during operation.

Table 2. Drag components for the Baseline and NLF Design configurations. The NLF Design configuration is analyzed both with appropriately predicted extents of laminar flow and with fully turbulent flow (no laminar flow).

\begin{tabular}{|c|c|c|c|}
\hline Configuration & $\mathbf{C}_{\mathbf{D}}$ [counts] & $\mathbf{C}_{\mathbf{D}, \text { viscous }}$ [counts] & $\mathbf{C}_{\mathbf{D}, \text { pressure }}$ [counts] \\
\hline NJWB Baseline & 82.6 & 43.4 & 39.2 \\
\hline NLF Design & 78.4 & 37.1 & 41.3 \\
\hline NLF Design, Fully Turbulent & 85.9 & 43.7 & 42.2 \\
\hline
\end{tabular}

USM3D predicts 2.1 counts of pressure drag penalty when going to a configuration that can support NLF (baseline to design). The designed laminar boundary layer provided 6.3 counts viscous drag savings in USM3D. Overall, USM3D predicts 4.2 drag counts of aerodynamic benefit when comparing the NLF Design to the Baseline, which represents approximately $5 \%$ of the total baseline drag. If all laminar flow were to be lost during operation, the vehicle would suffer a 3.3 drag count penalty from the NJWB Baseline or a 7.5 count penalty from the NLF Design with laminar flow.

Further comparing of the USM3D and Cart3D results shows that the drag components predicted by each tool (including the independent skin friction drag calculator developed to supplement Cart3D) provided similar performance predictions. USM3D estimated 2.1 counts of pressure drag penalty and Cart3D estimated 3.4 counts of inviscid drag penalty from Baseline to NLF Design. A likely source of the difference comes from Cart3D not accounting for the profile drag (which is included in USM3D pressure drag). Similarly, USM3D and the skin friction drag calculator used with Cart3D show a viscous drag benefit of 6.3 drag counts and 6.4 drag counts, respectively. Overall, it appears that Cart3D only slightly underestimates the potential drag benefit from utilizing NLF in this design, further validating the inviscid flow solver package as a useful initial design tool to reduce computational time and resources.

\section{Off-Design Analyses}

One concern with the NLF Design is that some resulting geometry features, such as the small leading-edge radii, may lead to significant losses at off-design conditions. Preliminary off-design evaluations of near-cruise and lowspeed, high-lift conditions are presented below, both of which were conducted using USM3D.

\section{Near-Cruise Analysis}

To address off-design cruise performance, the angle of attack of the vehicle is altered to attain a $\mathrm{C}_{\mathrm{L}}$ both $10 \%$ higher and lower than the design cruise $C_{L}$ of 0.1. All other flight conditions, such as Reynolds number and Mach number, are held constant. The solutions were obtained using USM3D with fully turbulent flow. The pressure distributions, examples of which are shown in Figure 18, were analyzed through the transition prediction process to determine transition fronts and then rerun in USM3D using the forced laminarization feature to account for the laminar flow up to the transition locations. 


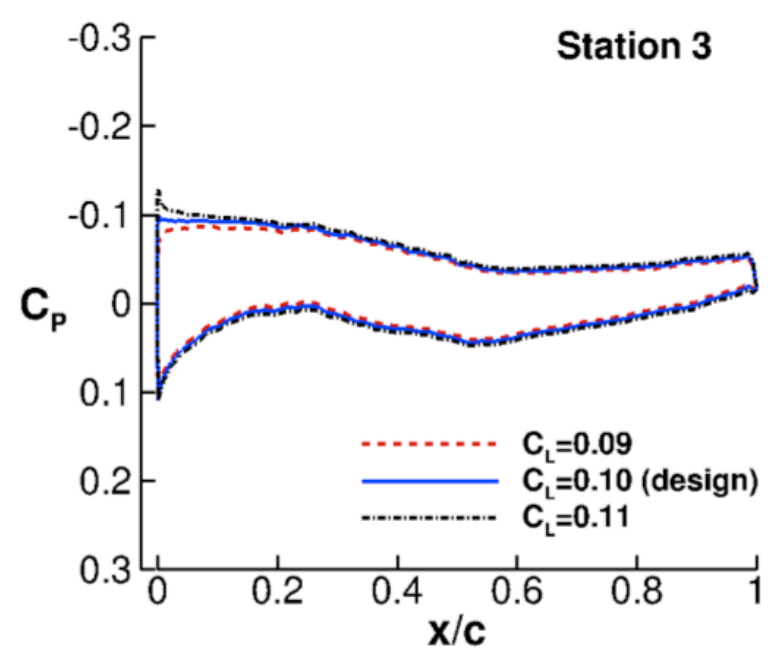

a) Station 3.

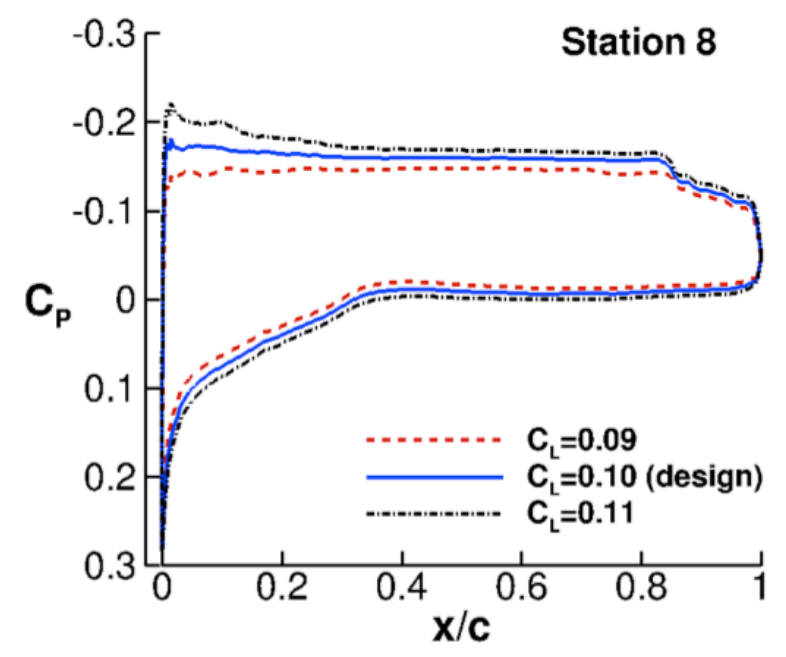

b) Station 8 .

Figure 18. Near-cruise pressure distributions at Stations 3 and 8. Compares $+/-10 \%$ design cruise lift.

At the design cruise $\mathrm{C}_{\mathrm{L}}$, a second iteration was performed with the USM3D trip feature turned on to evaluate the transition differences between a fully turbulent pressure distribution and a pressure distribution with a laminar flow region. The new pressure distribution with laminar flow accounted for were run through the transition prediction process, and then rerun in USM3D with the updated transition locations. Minimal changes were seen both in the flow solution and the transition front predicted using the fully turbulent pressure distribution and the pressure distribution with laminar flow region; thus it was determined a second iteration was not necessary for each nearcruise analysis point. The envelope curve of the modal stability analyses for TS and FB are shown in Figures 19 and 20 for Stations 3 and 8, respectively.

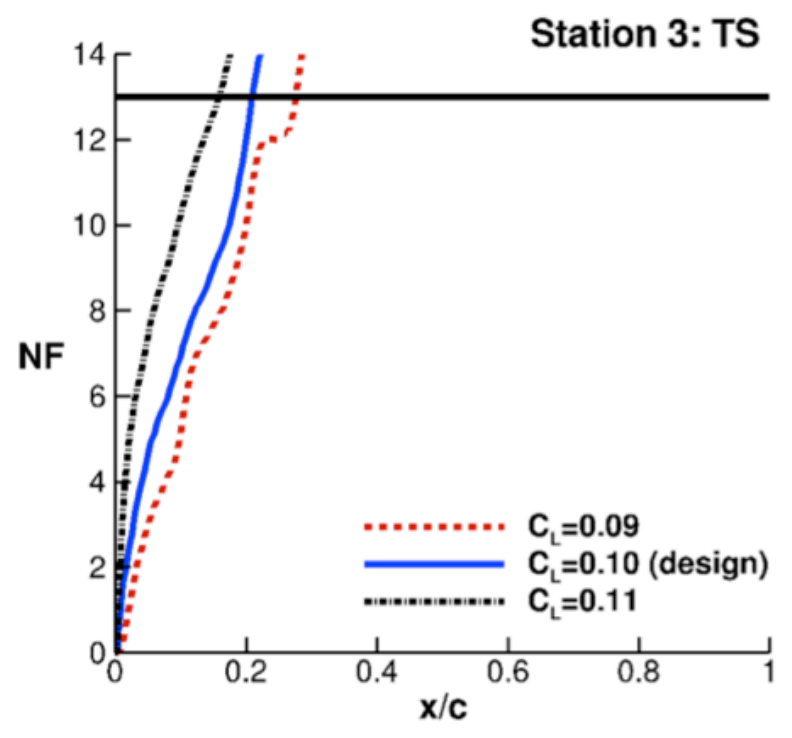

a) TS.

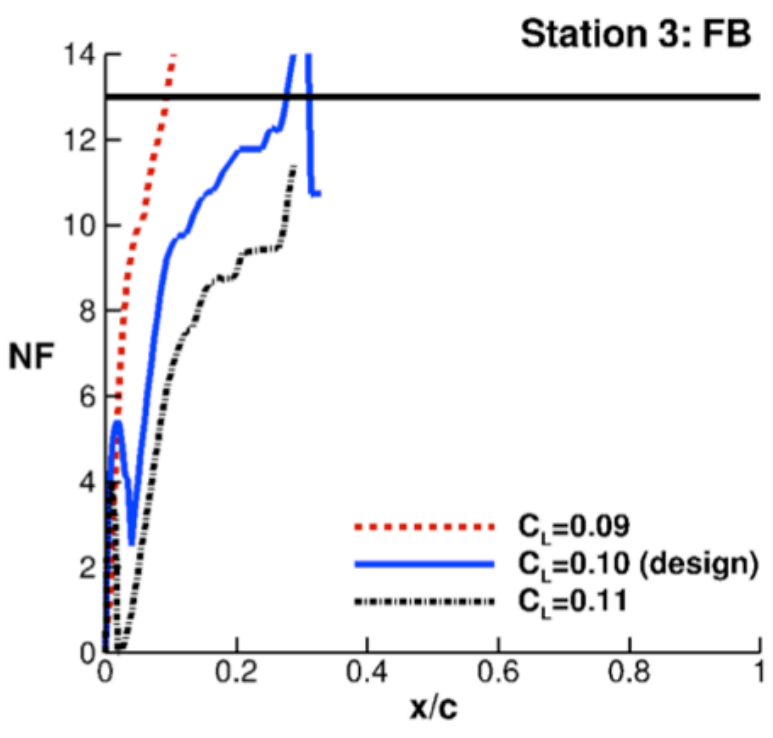

b) FB.

Figure 19. Envelope curves for the modal stability analyses of near-cruise pressure distributions at Station 3. 


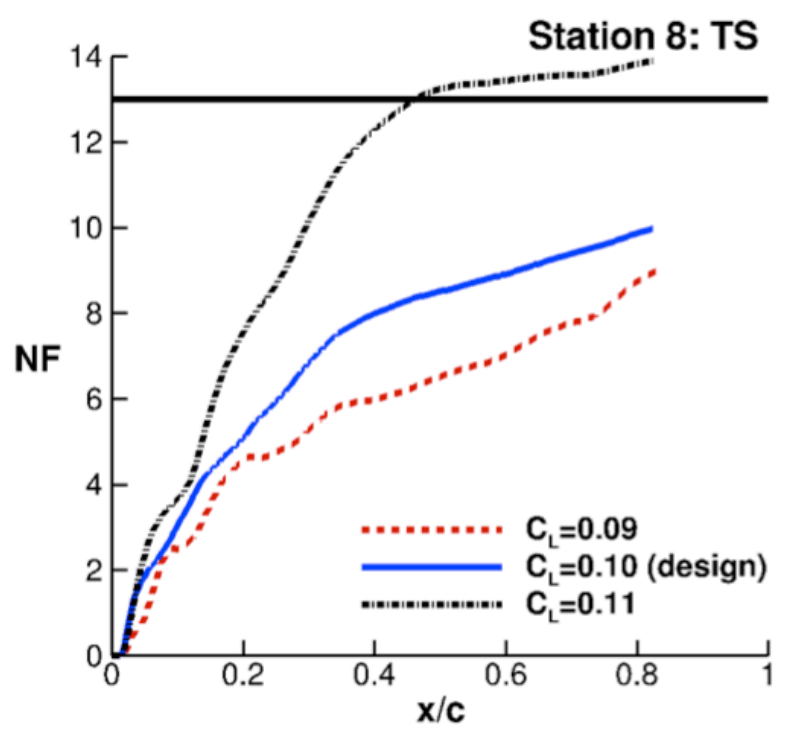

a) TS.

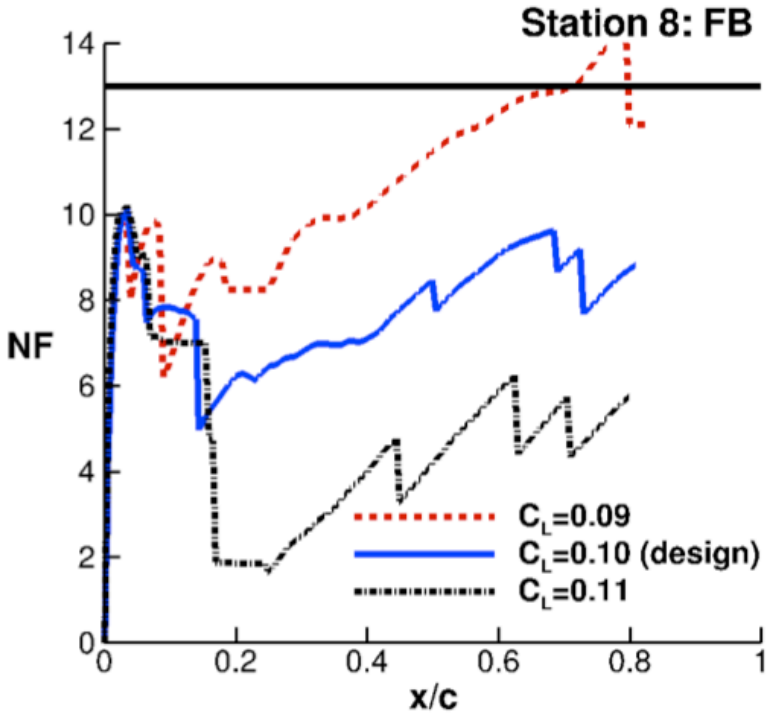

b) FB.

Figure 20. Envelope curves for the modal stability analyses of near-cruise pressure distributions at Station 8.

It was deduced from this off-design analysis that $\mathrm{FB}$ grows more rapidly and causes early transition at lower $\mathrm{C}_{\mathrm{L}}$, whereas TS is dominant at higher $\mathrm{C}_{\mathrm{L}}$. Changing angle of attack caused premature transition at most stations. The design cruise condition supported $40 \%$ laminar flow based on surface area, whereas reducing angle of attack (lower $\mathrm{C}_{\mathrm{L}}$ ) only had $19 \%$ laminar flow and increasing angle of attack (higher $\mathrm{C}_{\mathrm{L}}$ ) had $31 \%$ laminar flow. Figure 21 shows the transition front changes at the near-cruise conditions.

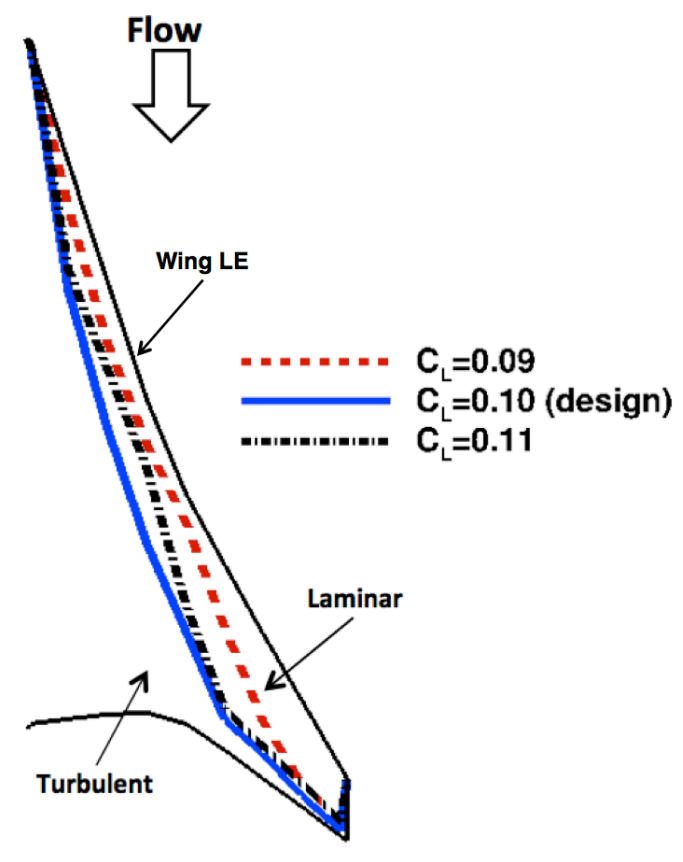

Figure 21. Near-cruise transition fronts showing laminar and turbulent flow regions on the planform of the wing.

The aerodynamic performance effects of losing laminar flow with changing angles of attack is illustrated in Figure 22, which shows a drag polar for both the NJWB Baseline and NLF Design. In addition, the NLF Design analyzed fully turbulent is plotted, which demonstrates the potential performance if all laminar flow were lost during 
operation. This plot indicates that some drag benefit remains throughout the off-design cruise range defined above, shown by the dashed horizontal lines.

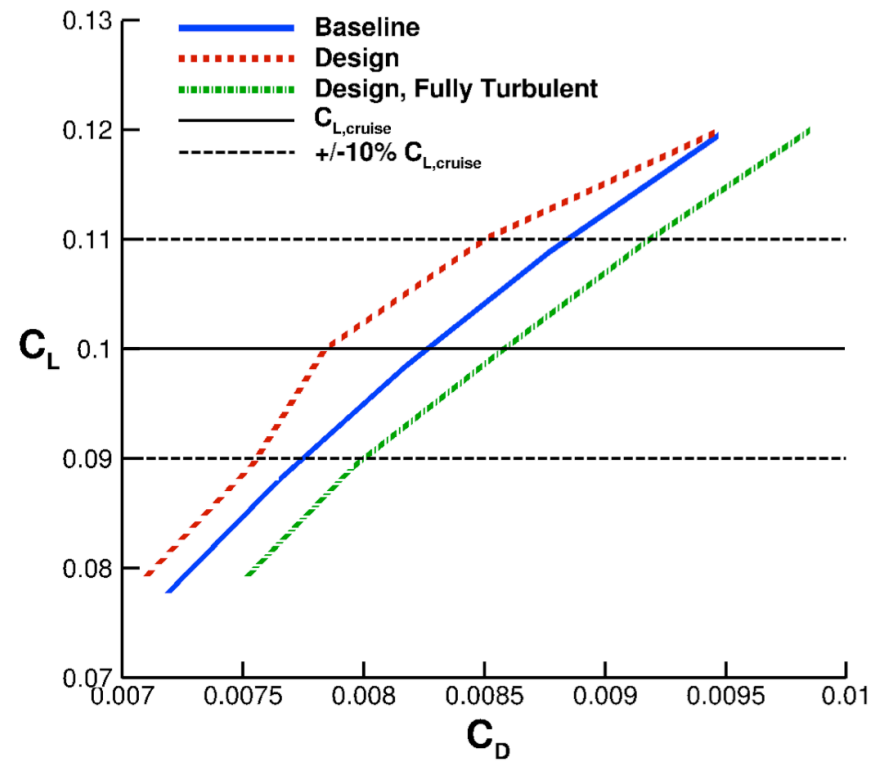

Figure 22. Cruise drag polar for Baseline and NLF Design configurations.

\section{Low-Speed, High-Lift Analysis}

The preliminary evaluation of low-speed, high-lift performance comparing the Baseline and NLF Design configurations was conducted using USM3D. Both geometries are assumed to have fully turbulent boundary layers with no flaps deployed and are evaluated at sea-level conditions at a Mach of 0.2 and angle of attack ranging from 10-25 degrees. New grids were generated for both configurations to perform this analysis that had a further refined leading edge and outer boundary consistent with low speed flow. The performance results for these conditions are compared in Figures 23 and 24.

Both the lift curve (Figure 23) and the drag polar (Figure 24) show negligible changes between the Baseline and Design geometries at the low-speed, high-lift conditions evaluated. The Design has a slight increase in drag at lower alphas (lower $C_{L}$ values) and a slight increase in lift at higher alphas. Further low-speed, high-lift evaluations, perhaps including high-lift devices, would be necessary to make any conclusions about the overall performance tradeoffs between the two configurations. However, the preliminary analysis suggests no significant change when going from Baseline to NLF Design.

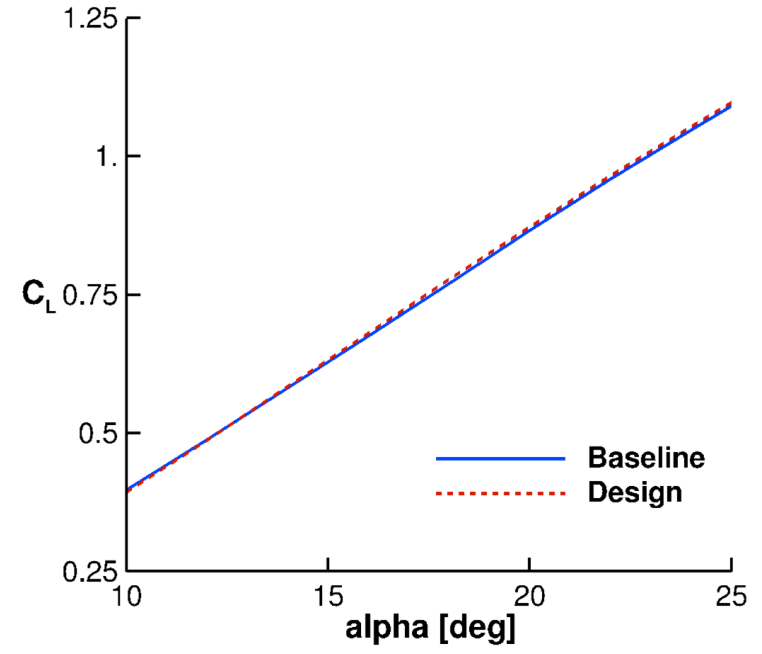

Figure 24. Lift curve comparing Baseline and Design at low-speed, high-lift conditions.

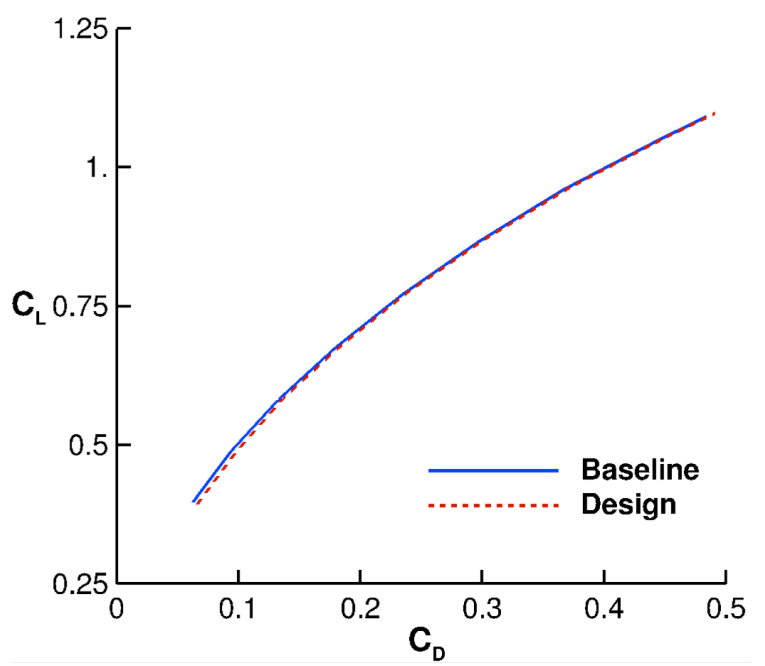

Figure 23. Drag polar comparing Baseline and Design at low-speed, high-lift conditions. 


\section{Attachment Line Contamination/Transition}

Successfully eliminating attachment line contamination and transition is essential to achieving NLF. As previously mentioned, Poll's criteria ${ }^{3}$ of $\operatorname{Re}_{\theta} \leq 100$ and $\mathrm{Re}_{\theta} \leq 235$ will eliminate $\mathrm{AL}$ contamination and transition, respectively. The CDISC leading-edge radius constraint was designed to provide a specified $\operatorname{Re}_{\theta}$ value at the leading edge of the wing station. At the time of design for the NJWB application, a leading-edge radius corresponding to a $\mathrm{Re}_{\theta} \leq 100$ was pursued. While the CDISC constraint successfully met the desired radius at the leading edge, it was later discovered that the more vital $\mathrm{Re}_{\theta}$ was the attachment point itself, which is not necessarily located at the leading edge. This realization led to an evaluation of attachment point surface curvature in an attempt to predict the state of the boundary layer along the AL.

Figure 25 shows an example of the initial surface curvature-based AL boundary layer investigation for Stations 3 and 8 . Negative $\mathrm{x} / \mathrm{c}$ represents the lower surface, positive $\mathrm{x} / \mathrm{c}$ represents the upper surface, and $\mathrm{x} / \mathrm{c}=0$ is the leading edge. These plots show the surface curvature normalized by local chord, and illustrate the high peak (i.e., small radius) at the leading edge added from the CDISC constraint; the peak values correspond to a $\operatorname{Re}_{\theta} \leq 100$. However, the $\mathrm{x} / \mathrm{c}$ region of high curvature is very small. In Figure 25, a horizontal line representing a curvature that corresponds to $\mathrm{Re}_{\theta} \leq 235$ is shown. Two black dashed vertical lines are plotted where this $\operatorname{Re}_{\theta} \leq 235$ limit intersects the curvature line, which represents the $\mathrm{x} / \mathrm{c}$ range that the attachment line must fall within to be on a curvature that would meet the $\mathrm{Re}_{\theta} \leq 235$ criterion for AL transition (note that meeting the AL contamination criterion is an even smaller $\mathrm{x} / \mathrm{c}$ range).

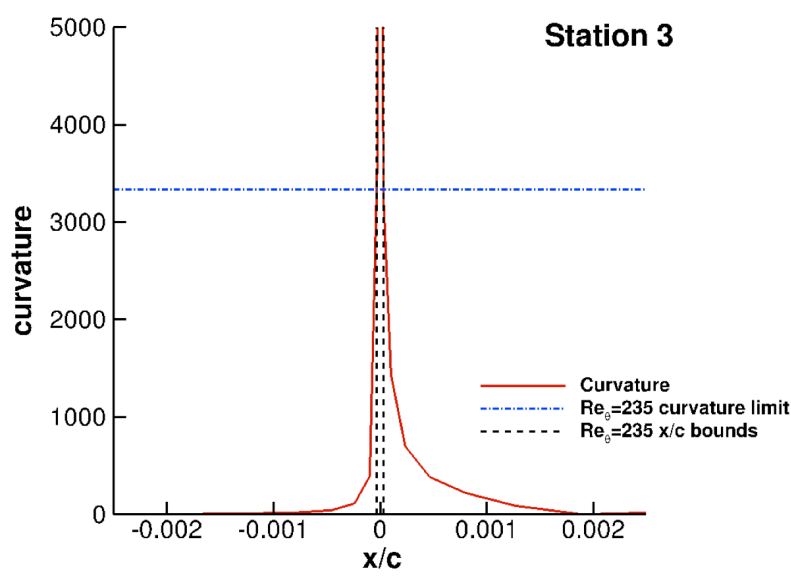

a) Station 3 .

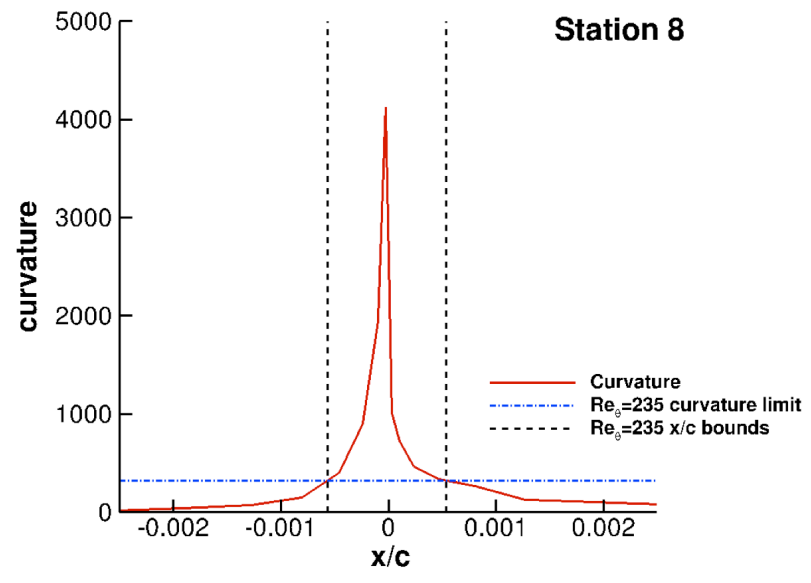

b) Station 8.

Figure 25. Curvature versus $\mathbf{x} / \mathbf{c}$ location plots showing leading-edge curvature and corresponding $\operatorname{Re}_{\theta}=235$ limits at Stations 3 and 8.

Figure 26 illustrates this information on the airfoil geometry, showing just the leading-edge region (first 1\% chord). In this figure, the blue triangles are the two $\mathrm{x} / \mathrm{c}$ locations determined by the black dashed vertical lines on the curvature plots for the $\mathrm{Re}_{\theta}=235$ limit. The attachment point, shown as a green square, must fall between the two blue triangles if the AL transition criterion is to be met. It was observed that the attachment point moved further aft on the lower surface and that most stations, especially inboard, would not meet the $\operatorname{Re}_{\theta} \leq 235$ requirement based on local curvature. 


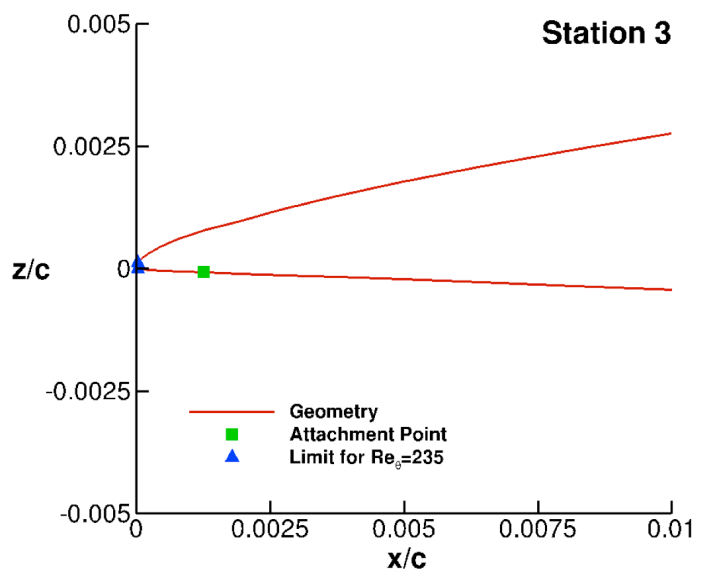

a) Station 3.

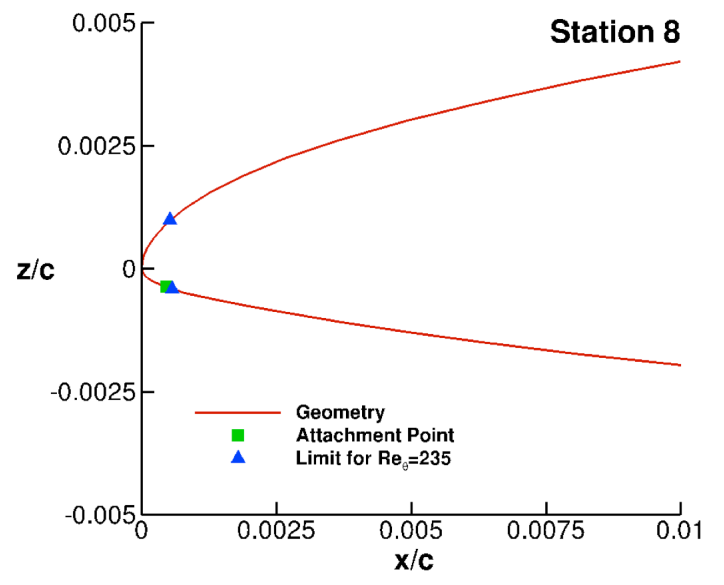

b) Station 8 .

Figure 26. Leading-edge geometry showing attachment point and $\operatorname{Re}_{\theta}=235$ limits based on curvature.

This curvature-based $\operatorname{Re}_{\theta}$ calculation was performed using the swept cylinder approximation ${ }^{4}$. As the present NLF design is not well represented by a swept cylinder, a flow-based $\mathrm{Re}_{\theta}$ calculation was desired. It was discovered that BLSTA3D provides an attachment point $\operatorname{Re}_{\theta}$ based on the pressure distribution. Figure 27 shows the spanwise distribution of the $\mathrm{AL} \mathrm{Re}_{\theta}$ from BLSTA3D and suggests the NLF Design is much closer to addressing AL contamination/transition based on Poll's criterion than the curvature-based evaluation shows. Similar BLSTA3D Re ${ }_{\theta}$ distributions show that at near-cruise off-design conditions, $\mathrm{Re}_{\theta}$ slightly increases but maintains the general characteristic of the NLF Design (i.e., $\operatorname{Re}_{\theta} \leq 235$ everywhere with $\mathrm{Re}_{\theta} \leq 100$ outboard).

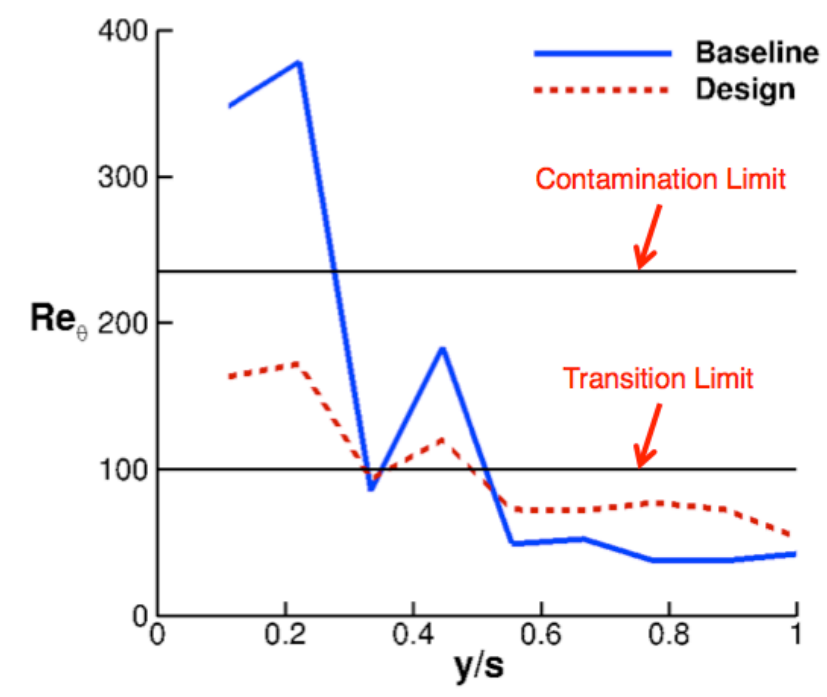

Figure 27. Baseline and Design spanwise $\operatorname{Re}_{\theta}$ distributions from BLSTA3D. Design Approach. At present, the NLF Design successfully damps AL transition, but requires additional effort to eliminate fuselage turbulent boundary layer contamination, as well as address any spanwise $\mathrm{C}_{\mathrm{P}}$ gradient concerns.

\section{Concluding Remarks}

Due to an increasing interest in returning to commercial supersonic flight, resources are being invested into developing quiet, cost-effective, and environmentally friendly aircraft. One drag-saving technology that would help realize this goal is the practical application of natural laminar flow (NLF) on supersonic aircraft. The research presented in this paper discusses a computationally based method that would allow the design of a supersonic transport to sustain significant regions of NLF. Maintaining a laminar boundary layer would provide significant skin 
friction drag savings, thus potentially decreasing the overall drag of the aircraft, which would translate to savings in fuel burn related costs and environmental concerns.

The NLF design methodology described in this paper operates on altering wing pressure distributions to provide pressure gradients that damp out dominant modal instability waves, including crossflow (CF), Tollmien-Schlichting (TS), and frequency-beta (FB). The computational tools used include: Cart3D and USM3D for flow solutions, BLSTA3D and LASTRAC for transition prediction, and CDISC as the design module. The effort described in this paper provide a status report on the attempt to answer three main questions relating to NLF design:

1) Can a target pressure distribution be defined that will support a significant region of laminar flow and meet other flow constraints (lift, pitching moment, shock strength, etc.)?

2) Can geometry be designed to match the target pressures with sufficient accuracy to largely obtain the target laminar flow region while matching geometry constraints (thickness, leading-edge radius, twist, etc.)?

3) Does the attachment line for the design fall within the bounds defined by Poll's criteria ${ }^{3}$ to avoid attachment line contamination/transition?

In order to answer these questions, a new CDISC constraint, SSNLF, was developed to prescribe a target pressure distribution that would support NLF. The SSNLF constraint incorporated relationships between modal instabilities and pressure gradients, which were balanced by internally adjusting parameters (such as $\mathrm{x} 1$ and UDF) to provide the maximum extent of laminar flow at a given wing station. The CDISC NLF design method was demonstrated on a generic Concorde-size supersonic transport. The configuration and the computational design method were developed as part of a collaborative effort between researchers at NASA and JAXA. A companion paper, Natural Laminar Flow Design for Wings with Moderate Sweep, discusses the efforts to apply the CDISC NLF design method in transonic flight conditions to the Common Research Model.

The example baseline configuration was originally redesigned using the inviscid flow solver Cart3D and the resulting configuration was analyzed to provide flow and boundary layer characteristics. During this initial design stage, it was determined that there is a theoretical target pressure that could support significant regions of laminar flow, effectively answering question 1. Additionally, the pressures and resulting transition locations matched closely between the target and design. In order to more confidently answer question 2, the Cart3D baseline and design geometries were converted to USM3D-compatible grids to be run using the full Navier-Stokes code. The USM3D flow and stability analysis results suggest that significant regions of laminar flow can be sustained at high transition Reynolds number $\left(\mathrm{Re}_{\mathrm{T}}\right)$ and leading-edge sweeps $\left(\Lambda_{\mathrm{LE}}\right)$. Figure 28 shows the computational data gathered using the CDISC NLF design method compared to the current boundary of where laminar flow control (LFC) is needed to sustain laminar flow. The results suggest that this configuration significantly expands the NLF boundary for supersonic transports.

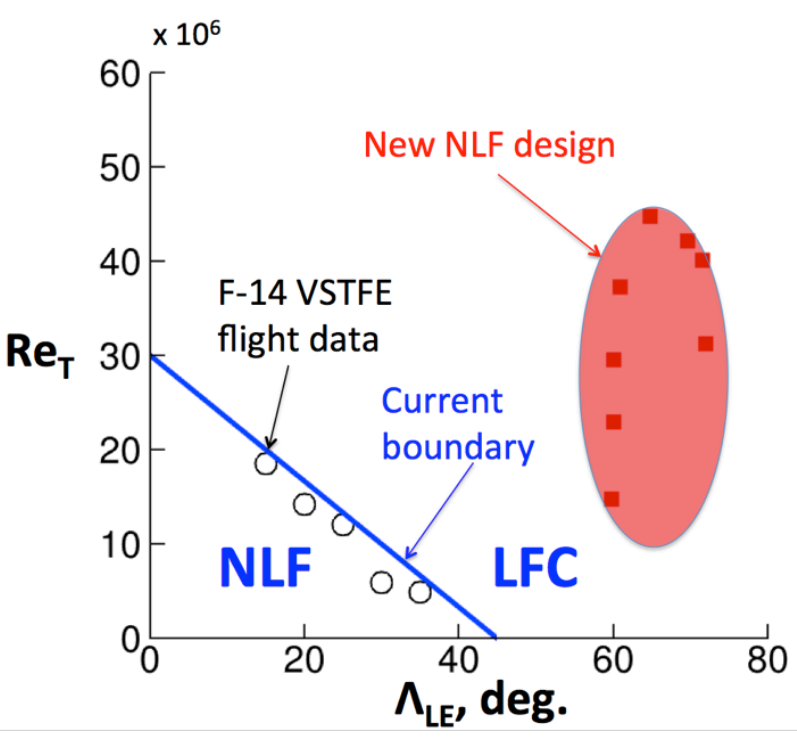

Figure 28. New NLF Design data shown with the current NLF-LFC boundary in terms of leading edge sweep $\left(\Lambda_{L E}\right)$ and transition Reynolds number $\left(\operatorname{Re}_{\mathrm{T}}\right)$.

The NLF Design configuration presented in this paper has a laminar boundary layer over approximately $40 \%$ of the upper surface of the wing by surface area. This region of NLF provides 6.3 counts of viscous drag savings from the Baseline configuration, while acquiring 2.1 counts of pressure drag penalty, which leads to a total drag decrease of 4.3 counts or approximately $5 \%$ of the wingbody configuration drag. The USM3D solutions confirmed that there is a realistic geometry that can support significant regions of laminar flow, effectively answering question 2. Additionally, after comparing the results from the USM3D solutions to the Cart3D solutions, it was determined that Cart3D is a valid initial design stage tool to save computational time and resources.

The NLF Design geometry was evaluated at two off-design conditions: near-cruise and low-speed, high-lift. The near-cruise analysis investigated the effects of operating the vehicle at $\pm 10 \%$ design cruise lift coefficient $\left(\mathrm{C}_{\mathrm{L}}=0.1\right)$. It was concluded that while losses in the total laminar flow occurred when changing angles of attack, there was still enough laminar flow to maintain a performance benefit over the Baseline configuration. However, if all laminar 
flow were lost during operation, the vehicle would suffer a 3.3 drag count penalty from the Baseline or a 7.5 drag count penalty from the NLF Design with laminar flow at the design cruise lift. The low-speed, high-lift analysis saw little change to the lift curve between the Baseline and NLF Design (with no laminar flow) configurations over the flight conditions evaluated (Mach $=0.2$, angle of attack $=10-25$ degrees). There was a shift in the drag polar curve showing that the fully turbulent NLF Design has higher drag for a given lift compared to the Baseline at the same low-speed, high-lift conditions.

To answer question 3 regarding attachment line (AL) concerns, Poll's criterion ${ }^{3}$ for avoiding AL contamination $\left(\operatorname{Re}_{\theta} \leq 100\right)$ and transition $\left(\operatorname{Re}_{\theta} \leq 235\right)$ were enforced. During the CDISC design process, a leading-edge radius constraint was implemented to ensure that the leading edge of the wing maintained a curvature corresponding to $\mathrm{Re}_{\theta}$ $\leq 100$. Upon closer inspection, it was determined that the attachment line was not necessarily at the leading edge, which raised concerns about the actual $\mathrm{AL} \mathrm{Re}_{\theta}$ values (instead of leading-edge $\mathrm{Re}_{\theta}$ values). A flow-based calculation provided by the boundary layer solver, BLSTA3D, shows that the attachment line maintains a $\operatorname{Re}_{\theta} \leq 235$ (therefore, avoiding AL transition) along the span of the wing. This holds true for near-cruise off-design conditions as well. However, AL contamination is not currently protected against, which suggests the need for either a redesign inboard (to obtain $\operatorname{Re}_{\theta} \leq 100$ at the attachment line) or a Gaster bump ${ }^{2}$ (to divert the turbulent fuselage boundary layer away from the leading edge). Ongoing research is being done to determine the best method to address AL contamination on this vehicle.

\section{Acknowledgments}

This research was funded by the NASA Commercial Supersonic Technology Project within the Advanced Air Vehicle Program. The work originated from a cooperative agreement between NASA and the Japan Aerospace Exploration Agency (JAXA) to conduct research on boundary layer transition at supersonic speeds. The authors would like to thank Dr. Mathias Wintzer for designing the baseline NJWB configuration, and the JAXA research team for their collaboration, especially Dr. Naoko Tokugawa and Dr. Hiroaki Ishikawa. Additionally, the authors would like to thank Dr. Meelan M. Choudhari and Dr. Chau-Lyan Chang of the NASA Langley Research Center for offering their support and expertise in boundary layer stability computations.

\section{References}

${ }^{1}$ Joslin, R. D., “Overview of Laminar Flow Control,” NASA TP-1998-208705, 1998.

${ }^{2}$ Gaster, M., “On the Flow Along Swept Leading Edges,” Aeronaut. Q., Vol. XVIII, pt. 2, 1967, pp. 165-184.

${ }^{3}$ Poll, D. I. A., "Some Observations of the Transition Process on the Windward Face of a Long Yawed Cylinder," J. Fluid Mech., Vol. 150, 1985, pp. 329-356.

${ }^{4}$ Garzon, G. A., and Matisheck, J. R., "Supersonic Testing of Natural Laminar Flow on Sharp Leading Edge Airfoils. Recent Experiments by Aerion Corporation,” AIAA 2012-3258, June 2012.

${ }^{5}$ Anderson, B. T., and Meyer, R. R., "Effects of Wing Sweep on In-Flight Boundary Layer Transition for a Laminar Flow Wing at Mach Numbers from 0.60 to 0.79 ," NASA TM-101701, 1990.

${ }^{6}$ Vermeersch, O., Yoshida, K., Ueda, Y., and Arnal, D., "Natural laminar flow wing for supersonic conditions: Wind tunnel experiments, flight test and stability computations," Progress in Aerospace Sciences, 2015.

${ }^{7}$ Ueda, Y., Yoshida, K., Matsushima, K., and Ishikawa, H., "Supersonic Natural-Laminar-Flow Wing-Design Concept at High-Reynolds-Number Conditions,” AIAA Journal, Vol. 52, No. 6, 2014, pp. 1294-1306.

${ }^{8}$ Melton, J. E., Berger, M. J., Aftosmis, M. J., and Wong, M. D., "3D Applications of a Cartesian Grid Euler Method," AIAA 95-0853, 1995.

${ }^{9}$ Frink, N.T., Pirzadeh, S.Z., Parikh, P.C., Pandya, M.J., and Bhat, M.K., "The NASA Tetrahedral Unstructured Software System," The Aeronautical Journal, Vol. 104, No. 1040, 2000, pp.491-499.

${ }^{10}$ Roe, P., "Characteristic Based Schemes for the Euler Equations," Annual Review of Fluid Mechanics, Vol. 18, 1986, pp. 337-365.

${ }^{11}$ Campbell, R. L., Campbell, M. L., and Streit, T., "Progress Toward Efficient Laminar Flow Analysis and Design,” AIAA 2011-3527, 2011.

${ }^{12}$ Chang, C.-L., "The Langley Stability and Transition Analysis Code (LASTRAC): LST, Linear and Nonlinear PSE for 2-D, Axisymmetric, and Infinite Swept Wing Boundary Layers," AIAA 2003-0974, 2003.

${ }^{13}$ Wie, Y.-S., "BLSTA: A Boundary Layer Code for Stability Analysis," NASA CR 4481, 1992.

${ }^{14}$ Campbell, R. L., "Efficient Viscous Design of Realistic Aircraft Configurations," AIAA-98-2539, 1998.

${ }^{15}$ Belisle, M. J., Roberts, M. W., Williams, T. C., Tufts, M. W., Tucker, A. A., Saric, W. S., and Reed, H. L., "A Transonic Laminar-Flow Wing Glove Flight Experiment: Overview and Design Optimization," AIAA 2012-2667, 2012. 